\title{
Similarity Measures between Temporal Complex Intuitionistic Fuzzy Sets and Application in Pattern Recognition and Medical Diagnosis
}

\author{
Mohammed M. Khalaf $\mathbb{D}^{1},{ }^{1}$ Sayer Obaid Alharbi, ${ }^{2}$ and Wathek Chammam $\mathbb{D}^{2,3}$ \\ ${ }^{1}$ Higher Institute of Engineering and Technology King Marriott, P.O. Box 3135, Egypt \\ ${ }^{2}$ Department of Mathematics, College of Science Al-Zulfi, Al-Majmaah University, P.O. Box 66, Al-Majmaah 11952, Saudi Arabia \\ ${ }^{3}$ Department of Mathematics, Faculty of Sciences of Gabès, Gabès University, Gabès, Tunisia \\ Correspondence should be addressed to Mohammed M. Khalaf; khalfmohammed2003@yahoo.com
}

Received 27 March 2019; Accepted 2 June 2019; Published 1 July 2019

Academic Editor: Francisco R. Villatoro

Copyright ( 2019 Mohammed M. Khalaf et al. This is an open access article distributed under the Creative Commons Attribution License, which permits unrestricted use, distribution, and reproduction in any medium, provided the original work is properly cited.

\begin{abstract}
This work addresses the issue of similarity measures between two temporal complex Atanassov's intuitionistic fuzzy sets, many measures of similarity between complex Atanassov's intuitionistic fuzzy sets. What was proposed before did not consider the abstention group influence, which may lead to counterintuitive results in some cases. A new structure of temporal complex Atanassov's intuitionistic fuzzy sets is obtained. This set is formally generalized from a conventional Atanassov's intuitionistic complex fuzzy sets. Here we analyze the limitations of the existing similarity measures. Then, a new similarity measure of temporal complex Atanassov's intuitionistic fuzzy sets is proposed and several numeric examples are given to demonstrate the validity of the proposed measure. Finally, the proposed similarity measure is applied to pattern recognition and medical diagnosis.
\end{abstract}

\section{Introduction}

Fuzzy set theory was conferred by Zadeh [1] to solve difficulties in dealing with uncertainties. Since then, the theories of fuzzy sets and fuzzy logic have been examined by many researchers to solve many real life problems involving ambiguous and uncertain environment. By adding a new component the idea of the concept of Atanassov's intuitionistic fuzzy set (AIFS) was introduced [2]. Applications of these sets have been broadly studied in other aspects such as image processing [3], multicriteria decision making [4], pattern recognition [5], etc. Buckley [6] and Nguyen et al. [7] combined complex numbers with fuzzy sets. On the other hand, the innovative complex fuzzy set is introduced. The complex fuzzy set is characterized by a membership function, $\mu_{\mathscr{A}}(x)$, whose range is not limited to $[0,1]$ but extended to the unit circle in the complex plane. Hence, $\mu_{\mathscr{A}}(x)$ is a complex-Valued function that assigns a grade of membership of the form $r_{\mathscr{A}}(x) e^{i \omega_{\mathscr{A}}(x)}, i=\sqrt{-1}$ to any element in the universe of discourse. The value of $\mu_{S}(x)$ is defined by the two variables, $r_{\mathscr{A}}(x)$ and $\omega_{\mathscr{A}}(x)$, both real-valued, with $\mu_{\mathscr{A}}(x) \epsilon$ $[0,1]$. Complex fuzzy set theory modifies the original concept of fuzzy membership by asserting that, at least in some instances, it is necessary to add a second dimension to the expression of membership. However, this added dimension does not alter the basic concept of fuzziness. Membership in a complex fuzzy set remains "as fuzzy" as membership in a traditional fuzzy set. The fuzziness of membership, i.e., the representation of membership as a value in the range $[0,1]$, is retained in complex fuzzy sets through the amplitude of the grade of membership, $r_{\mathscr{A}}(x)$. The novelty of complex fuzzy sets is manifested in the additional dimension of membership: the phase of the grade of membership, $\omega_{\mathscr{A}}(x)$. The properties of membership phase are discussed at length in this section. Ramot et al. [8,9] extended the range of membership to "unit circle in the complex plane", unlike others who limited the range to $[0,1]$. Omar $[10]$ studied similarity measures between temporal intuitionistic fuzzy sets. As the complex fuzzy membership grade is twodimensional (amplitude and phase), a complex fuzzy set can 


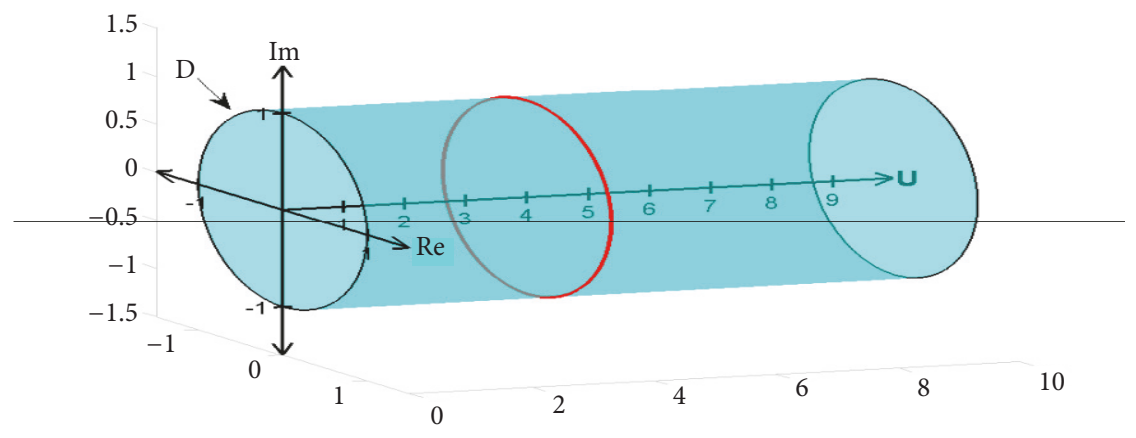

FIGURE 1: Complex fuzzy set defined in [11].

be visually represented by a three-dimensional graph where the universe of discourse is the third axis. Figure 1 shows the complex fuzzy set.

We divide the paper into four main sections. In the first section preliminaries and basic definitions, we provide some details about the complex fuzzy sets. In the second section, detail is given about the complex version of temporal complex intuitionistic fuzzy set, which is an extension of complex intuitionistic fuzzy set by adding the times and studied the correlation coefficient between two temporal complex intuitionistic fuzzy set. In the third section, details is given about similarity measures between other extensions of temporal complex intuitionistic fuzzy set and extend the method proposed by Chaira [12] for intuitionistic fuzzy set based on the Sugeno [13] and Omar [10] intuitionistic fuzzy generator. In the fourth section, we give application in pattern recognition, medical diagnosis, and topology.

\section{Preliminaries and Basic Definitions}

Definition 1 (see [8]). A complex fuzzy set (CFS) $\mathscr{A}$ defined on a universe $X$ is an object of the form $\mathscr{A}$ defined on a universe of discourse $X$ which is an object of the form

$$
\mathscr{A}=\left\{x, \mu_{\mathscr{A}}(x) e^{i \omega_{\mathscr{A}}(x)}: x \in X\right\}
$$

where $i=\sqrt{-1}, \mu_{\mathscr{A}}(x) \in[0,1]$, and $0 \leq \omega_{\mathscr{A}}(x) \leq 2 \pi$.

Definition 2 (see [2]). A complex intuitionistic fuzzy set (CIFS) $\mathscr{A}$ defined on a universe of discourse $X$ is an object of the form

$$
\mathscr{A}=\left\{x, \mu_{\mathscr{A}}(x) e^{i \alpha_{\mathscr{A}}(x)}, v_{\mathscr{A}}(x) e^{i \beta_{\mathscr{A}}(x)}: x \in X\right\},
$$

where $i=\sqrt{-1}, \mu_{\mathscr{A}}(x), v_{\mathscr{A}}(x) \in[0,1], \alpha_{\mathscr{A}}(x), \beta_{\mathscr{A}}(x) \in$ $[0,2 \pi]$, and $0 \leq \mu_{A}(x)+v_{A}(x) \leq 1$.

Definition 3 (see [14]). Let $\mathscr{A}$ and $\mathscr{B}$ be two CIFSs in $X$, where

$$
\begin{aligned}
& \mathscr{A}=\left\{x, \mu_{\mathscr{A}}(x) e^{i \alpha_{\mathscr{A}}(x)}, \nu_{\mathscr{A}}(x) e^{i \beta_{\mathscr{A}}(x)}: x \in X\right\} \\
& \mathscr{B}=\left\{x, \mu_{\mathscr{B}}(x) e^{i \alpha_{\mathscr{B}}(x)}, \nu_{\mathscr{B}}(x) e^{i \beta_{\mathscr{B}}(x)}: x \in X\right\}
\end{aligned}
$$

Then, $\mathscr{A} \cup \mathscr{B}$ is given as

$$
\begin{aligned}
\mathscr{A} & \cup \mathscr{B} \\
& =\left\{x, \mu_{\mathscr{A} \cup \mathscr{B}}(x) e^{i \alpha_{\mathscr{A} \cup \mathscr{B}}(x)}, v_{\mathscr{A} \cap \mathscr{B}}(x) e^{i \beta_{\mathscr{A} \cap \mathscr{B}}(x)}: x \in X\right\}
\end{aligned}
$$

where

$$
\begin{aligned}
& \mu_{\mathscr{A} \cup \mathscr{B}}(x) e^{i \alpha_{\mathscr{A} \backslash \mathscr{B}}(x)} \\
& =\left\{x,\left[\mu_{\mathscr{A}}(x) \vee v_{\mathscr{B}}(x)\right] e^{i\left\{\alpha_{\mathscr{A}}(x) \vee \alpha_{\mathscr{B}}(x)\right\}}\right\}, \\
& v_{\mathscr{A} \cap \mathscr{B}}(x) e^{i \alpha_{\mathscr{A} \cap \mathscr{B}}(x)} \\
& =\left\{x,\left[\nu_{\mathscr{A}}(x) \wedge v_{\mathscr{B}}(x)\right] e^{i\left\{\beta_{\mathscr{A}}(x) \wedge \beta_{\mathscr{B}}(x)\right\}}\right\} .
\end{aligned}
$$

Definition 4 (see [15]). Let $\mathscr{A}$ and $\mathscr{B}$ be two CIF-sets in $X$, where

$$
\begin{aligned}
& \mathscr{A}=\left\{x, \mu_{\mathscr{A}}(x) e^{i \alpha_{\mathscr{A}}(x)}, \nu_{\mathscr{A}}(x) e^{i \beta_{\mathscr{A}}(x)}: x \in X\right\} \\
& \mathscr{B}=\left\{x, \mu_{\mathscr{B}}(x) e^{i \alpha_{\mathscr{B}}(x)}, \nu_{\mathscr{B}}(x) e^{i \beta_{\mathscr{B}}(x)}: x \in X\right\} .
\end{aligned}
$$

Then, for all $x \in X$,

(1) $\mathscr{A} \subset \mathscr{B}$ if and only if $\mu_{\mathscr{A}}(x)<\mu_{\mathscr{B}}(x) ; \nu_{\mathscr{A}}(x)>\nu_{\mathscr{B}}(x)$. For amplitude terms and $\alpha_{\mathscr{A}}(x)<\alpha_{\mathscr{B}}(x), \beta_{\mathscr{A}}(x)>\beta_{\mathscr{B}}(x)$ for phase terms.

(2) $\mathscr{A}=\mathscr{B}$ if and only if $\mu_{\mathscr{A}}(x)=\mu_{\mathscr{B}}(x) ; \nu_{\mathscr{A}}(x)=\nu_{\mathscr{B}}(x)$. For amplitude terms and $\alpha_{\mathscr{A}}(x)=\alpha_{\mathscr{B}}(x), \beta_{\mathscr{A}}(x)=\beta_{\mathscr{B}}(x)$ for phase terms.

Definition 5 (see [16]). Let $\mathscr{A}$ and $\mathscr{B}$ be two CIFSs in $X$, where

$$
\begin{aligned}
& \mathscr{A}=\left\{x, \mu_{\mathscr{A}}(x) e^{i \alpha_{\mathscr{A}}(x)}, v_{\mathscr{A}}(x) e^{i \beta_{\mathscr{A}}(x)}: x \in X\right\} \\
& \mathscr{B}=\left\{x, \mu_{\mathscr{B}}(x) e^{i \alpha_{\mathscr{B}}(x)}, \nu_{\mathscr{B}}(x) e^{i \beta_{\mathscr{B}}(x)}: x \in X\right\}
\end{aligned}
$$

Then, for all $x \in X$, $X\}$

(1) $\mathscr{A} \cap \mathscr{B}=\left\{x, \mu_{\mathscr{A} \cap \mathscr{B}}(x) e^{i \alpha_{\mathscr{A} \cap \mathscr{B}}(x)}, v_{\mathscr{A} \cup \mathscr{B}}(x) e^{i \beta_{\mathscr{A} \cup \mathscr{B}}(x)}: x \in\right.$ 
where

$$
\begin{aligned}
& \mu_{\mathscr{A} \cap \mathscr{B}}(x) e^{i \alpha_{\mathscr{A} \cap \mathscr{B}}(x)} \\
& =\left\{x,\left[\mu_{\mathscr{A}}(x) \wedge \nu_{\mathscr{B}}(x)\right] e^{i\left\{\alpha_{\mathscr{A}}(x) \wedge \alpha_{\mathscr{B}}(x)\right\}}\right\}, \\
& v_{\mathscr{A} \cup \mathscr{B}}(x) e^{i \alpha_{\mathscr{A} \cup \mathscr{B}}(x)} \\
& \quad=\left\{x,\left[\nu_{\mathscr{A}}(x) \vee v_{\mathscr{B}}(x)\right] e^{i\left\{\beta_{\mathscr{A}}(x) \vee \beta_{\mathscr{B}}(x)\right\}}\right\} .
\end{aligned}
$$

(2) The complex fuzzy complement of $\mathscr{A}$, denoted by $\overline{\mathscr{A}}$, is specified by a function

$$
\begin{aligned}
\overline{\mathscr{A}} & =\left\{x,\left(1-\mu_{\mathscr{A}}(x)\right) e^{i\left(2 \pi-\alpha_{\mathscr{A}}(x)\right)},\left(1-\nu_{\mathscr{B}}(x)\right)\right. \\
& \left.\cdot e^{i\left(2 \pi-\beta_{\mathscr{A}}(x)\right)}: x \in X\right\}
\end{aligned}
$$
$X\}$.

(3) $\overline{1}=\left\{x,\left(e^{i(2 \pi)}, 0\right): x \in X\right\}$ and $\overline{0}=\left\{x,\left(0, e^{i(2 \pi)}\right): x \in\right.$

Example 6. Consider $X=\{a, b, c, d\}$. Let $\mathscr{A}, \mathscr{B}$ be a CIFsubset of $X$, as given by

$$
\begin{aligned}
\mathscr{A} & =\left(\frac{0.2 e^{i 1.3 \pi}, 0.4 e^{i 0.5 \pi}}{a}, \frac{1.0 e^{i 1.5 \pi}, 0.0 e^{i 0.5 \pi}}{b},\right. \\
& \left.\frac{0.7 e^{i 0.3 \pi}, 0.2 e^{i 1.5 \pi}}{c}, \frac{0.8 e^{i 1.1 \pi}, 0.1 e^{i 0.7 \pi}}{d}\right), \\
\mathscr{B}= & \left(\frac{0.2 e^{i 0.9 \pi}, 0.3 e^{i 0.4 \pi}}{a}, \frac{0.1 e^{i 0.2 \pi}, 0.3 e^{i 0.9 \pi}}{b},\right. \\
& \left.\frac{0.1 e^{i 0.1 \pi}, 0.2 e^{i 0.5 \pi}}{c}, \frac{0.5 e^{i 0.2 \pi}, 0.1 e^{i 0.5 \pi}}{d}\right) .
\end{aligned}
$$

Then

$$
\begin{gathered}
\mathscr{A} \cap \mathscr{B}=\left(\frac{0.2 e^{i 0.9 \pi}, 0.4 e^{i 0.5 \pi}}{a}, \frac{0.1 e^{i .2 \pi}, 0.3 e^{i 0.9 \pi}}{b},\right. \\
\left.\frac{0.1 e^{i 0.1 \pi}, 0.2 e^{i 1.5 \pi}}{c}, \frac{0.5 e^{i 0.2 \pi}, 0.1 e^{i 0.7 \pi}}{d}\right) \\
\overline{\mathscr{A}}=\left(\frac{0.8 e^{i 0.7 \pi}, 0.6 e^{i 1.5 \pi}}{a}, \frac{0,1.0 e^{i 1.5 \pi}}{b},\right. \\
\left.\frac{0.3 e^{i 1.7 \pi}, 0.8 e^{i 0.5 \pi}}{c}, \frac{0.2 e^{i 0.9 \pi}, 0.9 e^{i 1.3 \pi}}{d}\right) . \\
\mathscr{A} \cup \mathscr{B}=\left(\frac{0.2 e^{i 1.3 \pi}, 0.3 e^{i 0.4 \pi}}{a}, \frac{1.0 e^{i 1.5 \pi}, 0.0 e^{i 0.5 \pi}}{b},\right. \\
\left.\frac{0.7 e^{i 0.3 \pi}, 0.2 e^{i 0.5 \pi}}{c}, \frac{0.8 e^{i 1.1 \pi}, 0.1 e^{i 05 \pi}}{d}\right) .
\end{gathered}
$$

And $\mathscr{A} \not \subset \mathscr{B}$ and $\mathscr{A} \neq \mathscr{B}$.

\section{Temporal Complex Intuitionistic Fuzzy Set}

Definition 7. Let $X$ be a universe, $T$ be a nonempty set of time moments, and $\mathscr{A} \subseteq X$. A temporal complex intuitionistic fuzzy set (TCIFS) $\mathscr{A}$ defined on a universe of discourse $X$ is an object of the form

$$
\begin{gathered}
\mathscr{A}(T)=\left\{\left((x, t), \mu_{\mathscr{A}}(x, t) e^{i \alpha_{\mathscr{A}}(x, t)}, v_{\mathscr{A}}(x, t) e^{i \beta_{\mathscr{A}}(x, t)}\right) \mid\right. \\
(x, t) \in X \times T\},
\end{gathered}
$$

where $\mu_{\mathscr{A}}: \mathscr{A} \times T \longrightarrow[0,1]$ and $\nu_{\mathscr{A}}: \mathscr{A} \times T \longrightarrow$ $[0,1]$ such that $0 \leq \mu_{\mathscr{A}}(x, t) e^{i \alpha_{\mathscr{A}}(x, t)}+\nu_{\mathscr{A}}(x, t) e^{i \beta_{\mathscr{A}}(x, t)} \leq 1$, $\mu_{A}(x, t)$, and $v_{A}(x, t)$ being the degrees of membership and nonmembership, respectively, of the element $x \in X$ at the moment $t \in T$. And $\alpha_{\mathscr{A}}(x, t), \beta_{\mathscr{A}}(x, t) \in[0,2 \pi]$ at the moment $t \in T$, where $i=\sqrt{-1}$.

The hesitation degree of a TCIFS $\mathscr{A}$ is defined by $\pi_{\mathscr{A}}: 1-\mu_{\mathscr{A}}(x, t)-v_{\mathscr{A}}(x, t)$ such that $0 \leq \pi_{\mathscr{A}}(x, t) \leq 1$ for each $(x, t) \in \mathscr{A} \times T$. For brevity we will write $\mathscr{A}$ instead of $\mathscr{A}(T)$ when this does not cause confusions.

Example 8. Suppose that $X$ is a universal, with respect to the time set $T_{1}=\{t \mid t=6 k, k=0,1, \ldots, 100\}, T_{2}=\{t \mid t=$ $2 k, k=0,1, \ldots, 100\}$, and $\alpha_{\mathscr{A}}(x, t)=\beta_{\mathscr{A}}(x, t)=e^{i 2 \pi}$. Then, TCIFSs $\mathscr{A}, \mathscr{B}$ are defined by

$$
\begin{aligned}
& \mathscr{A}\left(T_{1}\right)(x)= \begin{cases}\left(\frac{1}{2}, \frac{1}{4}\right) & t=4 k, k=1, \ldots, 100 \\
\left(\frac{1}{9}, \frac{3}{5}\right) & t=4 k+2, k=1, \ldots, 100\end{cases} \\
& \mathscr{B}\left(T_{2}\right)(x)= \begin{cases}\left(\frac{2}{3}, \frac{1}{6}\right) & t=3 k, k=1, \ldots, 100 \\
\left(\frac{3}{8}, \frac{1}{5}\right) & t=3 k+1, k=1, \ldots, 100 \\
\left(\frac{7}{11}, \frac{2}{9}\right) & t=3 k+2, k=1, \ldots, 100\end{cases}
\end{aligned}
$$

Example 9. Suppose $X=\left\{x_{1}, x_{2}, x_{3}\right\}$ with respect to the time set $T=\left\{t_{1}, t_{2}, t_{3}\right\}$. Then, the details of a TCIFS $\mathscr{A}$ are explained in Tables 1,2 , and 3

Definition 10. Let $\mathscr{A}\left(T_{1}\right)$ and $\mathscr{B}\left(T_{2}\right)$ be two TCIFSs. Then

$$
\begin{aligned}
& \mathscr{A}\left(T_{1}\right) \cap \mathscr{B}\left(T_{2}\right)=\{(x, \\
& \min \left(\bar{\mu}_{\mathscr{A}}(x, t) e^{i \alpha_{\mathscr{A}}(x, t)}, \bar{\mu}_{\mathscr{B}}(x, t) e^{i \alpha_{\mathscr{B}}(x, t)}\right), \\
& \max \left(\bar{\nu}_{\mathscr{A}}(x, t) e^{i \beta_{\mathscr{A}}(x, t)}, \bar{v}_{\mathscr{B}}(x, t) e^{i \beta_{\mathscr{B}}(x, t)}\right):(x, t) \in X \\
& \quad \times\left(T_{1} \cup T_{2}\right\} \\
& \mathscr{A}\left(T_{1}\right) \cup \mathscr{B}\left(T_{2}\right)=\{(x, \\
& \quad \max \left(\bar{\mu}_{\mathscr{A}}(x, t) e^{i \alpha_{\mathscr{A}}(x, t)}, \bar{\mu}_{\mathscr{B}}(x, t) e^{i \alpha_{\mathscr{B}}(x, t)}\right), \\
& \min \left(\bar{\nu}_{\mathscr{A}}(x, t) e^{i \beta_{\mathscr{A}}(x, t)}, \bar{v}_{\mathscr{B}}(x, t) e^{i \beta_{\mathscr{B}}(x, t)}\right):(x, t) \in X \\
& \quad \times\left(T_{1} \cup T_{2}\right\}
\end{aligned}
$$


TABLE 1: TIFS $\mathscr{A}$.

\begin{tabular}{lccc}
\hline & $t_{1}$ & $t_{2}$ & $t_{3}$ \\
\hline$x_{1}$ & $(0.2,0.1)$ & $(0.1,0.6)$ & $(0.3,0.5)$ \\
\hline$x_{2}$ & $(0.6,0.1)$ & $(0.1,0.9)$ & $(0.6,0.4)$ \\
\hline$x_{3}$ & $(0.7,0.1)$ & $(0.1,0.7)$ & $(0.8,0.5)$ \\
\hline
\end{tabular}

TABLE 2: $\alpha_{\mathscr{A}}(x, t)=\beta_{\mathscr{A}}(x, t)$.

\begin{tabular}{lccc}
\hline & $t_{1}$ & $t_{2}$ & $t_{3}$ \\
\hline$x_{1}$ & $e^{i(\pi / 2)}$ & $e^{i \pi}$ & $e^{i 2 \pi}$ \\
\hline$x_{2}$ & $e^{i 2 \pi}$ & $e^{i(\pi / 2)}$ & $e^{i(\pi / 2)}$ \\
\hline$x_{3}$ & $e^{i(3 \pi / 2)}$ & $e^{i(3 \pi / 2)}$ & $e^{i(\pi / 2)}$ \\
\hline
\end{tabular}

TAble 3: TCIFS $\mathscr{A}$.

\begin{tabular}{cccc}
\hline & $t_{1}$ & $t_{2}$ & $t_{3}$ \\
\hline$x_{1}$ & $(0.2 i, 0.1 i)$ & $(-0.1,-0.6)$ & $(0.3,0.5)$ \\
\hline$x_{2}$ & $(0.6,0.1)$ & $(0.1 i, 0.9 i)$ & $(0.6 i, 0.4 i)$ \\
\hline$x_{3}$ & $(-0.7 i,-0.1 i)$ & $(-0.1 i,-0.7 i)$ & $(0.8 i, 0.5 i)$ \\
\hline
\end{tabular}

$$
\begin{aligned}
& \overline{\mathscr{A}}\left(T_{1}\right)=\{(x, \\
& \quad\left(\bar{\nu}_{\mathscr{A}}(x, t) e^{i\left(2 \pi-\beta_{\mathscr{A}}(x)\right)}, \bar{\mu}_{\mathscr{A}}(x, t) e^{i\left(2 \pi-\alpha_{\mathscr{A}}(x)\right)},\right):(x, t) \\
& \left.\quad \in X \times T_{1}\right\},
\end{aligned}
$$

where

$$
\begin{aligned}
& \bar{\mu}_{\mathscr{A}}(x, t)= \begin{cases}\mu_{\mathscr{A}}(x, t) & t \in T_{1} \\
0 & t \in T_{2}-T_{1}\end{cases} \\
& \bar{\nu}_{\mathscr{A}}(x, t)= \begin{cases}v_{\mathscr{A}}(x, t) & t \in T_{1} \\
1 & t \in T_{2}-T_{1}\end{cases} \\
& \bar{\mu}_{\mathscr{B}}(x, t)= \begin{cases}\mu_{\mathscr{B}}(x, t) & t \in T_{1} \\
0 & t \in T_{2}-T_{1}\end{cases} \\
& \bar{v}_{\mathscr{B}}(x, t)= \begin{cases}v_{\mathscr{B}}(x, t) & t \in T_{1} \\
1 & t \in T_{2}-T_{1}\end{cases}
\end{aligned}
$$

Definition 11. We define the following two operators $f$ and $g$ over a TCIFS $\mathscr{A}$ :

$$
\begin{gathered}
f(\mathscr{A}(T))=\left\{\left(x,\left\{\left(x, \frac{\max }{t \in T} \mu_{\mathscr{A}}(x, t) e^{i \alpha_{\mathscr{A}}(x, t)}, \frac{\min }{t \in T}\right.\right.\right.\right. \\
\left.\left.\left.\cdot \nu_{\mathscr{A}}(x, t) e^{i \beta_{\mathscr{A}}(x, t)}\right)\right\}:(x, t) \in X \times T\right\}
\end{gathered}
$$

$$
\begin{aligned}
& h(\mathscr{A}(T))=\left\{\left(x,\left\{\left(x, \frac{\max }{t \in X} \mu_{\mathscr{A}}(x, t) e^{i \alpha_{\mathscr{A}}(x, t)}, \frac{\min }{t \in X}\right.\right.\right.\right. \\
& \left.\left.\left.\cdot v_{\mathscr{A}}(x, t) e^{i \beta_{\mathscr{A}}(x, t)}\right)\right\}:(x, t) \in X \times T\right\} \\
& g(\mathscr{A}(T))=\left\{\left(x,\left\{\left(x, \frac{\min }{t \in T} \mu_{\mathscr{A}}(x, t) e^{i \alpha_{\mathscr{A}}(x, t)}, \frac{\max }{t \in T}\right.\right.\right.\right. \\
& \left.\left.\left.\cdot v_{\mathscr{A}}(x, t) e^{i \beta_{\mathscr{A}}(x, t)}\right)\right\}:(x, t) \in X \times T\right\} \\
& l(\mathscr{A}(T))=\left\{\left(x,\left\{\left(x, \frac{\min }{t \in X} \mu_{\mathscr{A}}(x, t) e^{i \alpha_{\mathscr{A}}(x, t)}, \frac{\max }{t \in X}\right.\right.\right.\right. \\
& \left.\left.\left.\cdot v_{\mathscr{A}}(x, t) e^{i \beta_{\mathscr{A}}(x, t)}\right)\right\}:(x, t) \in X \times T\right\}
\end{aligned}
$$

Theorem 12. $f(\mathscr{A}(T))$ and $g(\mathscr{A}(T))$ are TCIFSs.

Proof. Suppose that

$$
\frac{\max }{t \in T} \mu_{\mathscr{A}}(x, t) e^{i \alpha_{\mathscr{A}}(x, t)}=\mu_{\mathscr{A}}\left(x, t_{1}\right) e^{i \alpha_{\mathscr{A}}\left(x, t_{1}\right)},
$$

for some $t_{1} \in T$,

and

$$
\frac{\min }{t \in T} \nu_{\mathscr{A}}(x, t) e^{i \alpha_{\mathscr{A}}(x, t)}=\nu_{\mathscr{A}}\left(x, t_{2}\right) e^{i \alpha_{\mathscr{A}}\left(x, t_{2}\right)},
$$

for some $t_{2} \in T$

Therefore,

$$
\begin{aligned}
& v_{\mathscr{A}}\left(x, t_{2}\right) e^{i \beta_{\mathscr{A}}\left(x, t_{2}\right)} \leq v_{A}\left(x, t_{1}\right) e^{i \beta_{\mathscr{A}}\left(x, t_{1}\right)} \text {. And } \\
& \frac{\max }{t \in T} \mu_{\mathscr{A}}(x, t) e^{i \alpha_{\mathscr{A}}(x, t)}+\frac{\min }{t \in T} v_{A}(x, t) e^{i \alpha_{\mathscr{A}}(x, t)} \\
& \quad=\mu_{\mathscr{A}}\left(x, t_{1}\right) e^{i \alpha_{\mathscr{A}}\left(x, t_{1}\right)}+v_{A}\left(x, t_{2}\right) e^{i \alpha_{\mathscr{A}}\left(x, t_{2}\right)} \\
& \quad \leq \mu_{\mathscr{A}}\left(x, t_{1}\right) e^{i \alpha_{\mathscr{A}}\left(x, t_{1}\right)}+v_{A}\left(x, t_{1}\right) e^{i \beta_{\mathscr{A}}\left(x, t_{1}\right)} \leq 1
\end{aligned}
$$

Then $f(\mathscr{A}(T))$ is TCIFSs. Also, by the same fashion $g(\mathscr{A}(T))$ are TCIFSs.

Theorem 13. For every TCIFS $\mathscr{A}(T)$,
(1) $f(f(\mathscr{A}(T)))=f(\mathscr{A}(T))$;
(2) $g(g(\mathscr{A}(T)))=g(\mathscr{A}(T))$;
(3) $f(g(\mathscr{A}(T)))=g(\mathscr{A}(T))$;
(4) $g(f(\mathscr{A}(T)))=f(\mathscr{A}(T))$.

Proof. The proof is obvious.

Theorem 14. For every TCIFS $\mathscr{A}(T)$,

(1) $h(f(\mathscr{A}(T)))=f(h(\mathscr{A}(T)))$;

(2) $l(g(\mathscr{A}(T)))=g(l(\mathscr{A}(T)))$. 
Proof. (1)

$$
\begin{aligned}
& h(f(\mathscr{A}(T)))=\left\{\left(x,\left\{\left(x, \frac{\max }{t \in X} \frac{\max }{t \in T} \mu_{\mathscr{A}}(x, t)\right.\right.\right.\right. \\
& \left.\left.\cdot e^{i \alpha_{\mathscr{A}}(x, t)}, \frac{\min }{t \in X} \frac{\min }{t \in T} v_{\mathscr{A}}(x, t) e^{i \beta_{\mathscr{A l}}(x, t)}\right)\right\}:(x, t) \\
& \in X \times T\}=\left\{\left(x,\left\{\left(x, \frac{\max }{t \in T} \frac{\max }{t \in X} \mu_{\mathscr{A}}(x, t) e^{i \alpha_{\mathscr{A}}(x, t)},\right.\right.\right.\right. \\
& \left.\left.\left.\frac{\min }{t \in T} \frac{\min }{t \in X} v_{\mathscr{A}}(x, t) e^{i \beta_{\mathscr{A}}(x, t)}\right)\right\}:(x, t) \in X \times T\right\} \\
& =f(h(\mathscr{A}(T)))
\end{aligned}
$$

(2) By the same fashion, one has the following.

Definition 15. Let $\mathscr{A}$ and $\mathscr{B}$ be two TCIFSs defined on the universe of discourse $X=\left\{x_{1}, x_{2}, x_{3}, \ldots, x_{n}\right.$ and the time moments $T=\left\{t_{1}, t_{2}, t_{3}, \ldots, t_{m}\right\}$. The correlation coefficient of $\mathscr{A}$ and $\mathscr{B}$ is given by

$$
k(\mathscr{A}, \mathscr{B})=\frac{C(\mathscr{A}, \mathscr{B})}{\sqrt{T(\mathscr{A}) T(\mathscr{B})}}
$$

where

$$
\begin{aligned}
C & (\mathscr{A}, \mathscr{B}) \\
& =\sum_{i=1}^{n} \sum_{j=1}^{m}\left(\mu_{\mathscr{A}}\left(x_{i}, t_{i}\right) e^{i \alpha_{\mathscr{A}}(x, t)} \mu_{\mathscr{B}}\left(x_{i}, t_{i}\right) e^{i \alpha_{\mathscr{B}}(x, t)}\right. \\
& \left.+v_{\mathscr{A}}\left(x_{i}, t_{i}\right) e^{i \beta_{\mathscr{A}}(x, t)} v_{\mathscr{B}}\left(x_{i}, t_{i}\right) e^{i \beta_{\mathscr{B}}(x, t)}\right)
\end{aligned}
$$

is the correlation of two TCIFSs $\mathscr{A}$ and $\mathscr{B}$, and

$$
\begin{aligned}
T(\mathscr{A}) & =\sum_{i=1}^{n} \sum_{j=1}^{m}\left(\mu_{\mathscr{A}}{ }^{2}\left(x_{i}, t_{i}\right) e^{i\left(2 \pi-\alpha_{\mathscr{A}}(x)\right)}\right. \\
+v_{\mathscr{A}} & \left.{ }^{2}\left(x_{i}, t_{i}\right) e^{i\left(2 \pi-\beta_{\mathscr{A}}(x)\right)}\right), \\
T(\mathscr{B}) & =\sum_{i=1}^{n} \sum_{j=1}^{m}\left(\mu_{\mathscr{B}}^{2}\left(x_{i}, t_{i}\right) e^{i\left(2 \pi-\alpha_{\mathscr{B}}(x, t)\right)}\right. \\
& \left.+\nu_{\mathscr{B}}{ }^{2}\left(x_{i}, t_{i}\right) e^{i\left(2 \pi-\beta_{\mathscr{S}}(x)\right)}\right)
\end{aligned}
$$

are the information temporal complex intuitionistic energies of $\mathscr{A}$ and $\mathscr{B}$, respectively.

Example 16. Suppose that $X=\left\{x_{1}, x_{2}, x_{3}\right\}$ with respect to the time set $T=\left\{t_{1}, t_{2}, t_{3}\right\}$. The details of a TCIFS $\mathscr{A}(T)$ are explained in Table 4 , Table 5 explained TCIFS $\mathscr{B}(T)$, and Table 6 explained the correlation coefficient $k(\mathscr{A}, \mathscr{B})$ between TCIFS $\mathscr{A}(T)$ and TCIFS $\mathscr{B}(T)$.

Proposition 17. Let $\mathscr{A}\left(T_{1}\right)$ and $\mathscr{B}\left(T_{2}\right)$ be two TCIFS. Then

(1) $T(\mathscr{A})=T(\overline{\mathscr{A}})$;

(2) If $\alpha_{\mathscr{A}}(x, t)=2 \pi$ and $\beta_{\mathscr{A}}(x, t)=2 \pi$, then, $C(\mathscr{A}, \mathscr{A})=$ $T(\mathscr{A})$;

(3) $C(\mathscr{A}, \mathscr{B})=C(\mathscr{B}, \mathscr{A})$.
TABLE 4: TCIFS $\mathscr{A}(T)$.

\begin{tabular}{cccc}
\hline & $t_{1}$ & $t_{2}$ & $t_{3}$ \\
\hline$x_{1}$ & $(0.2 i, 0.3 i)$ & $(-0.1 i,-0.3 i)$ & $(0.3 i, 0.5 i)$ \\
\hline$x_{2}$ & $(0.6,0.1)$ & $(0.1 i, 0.9 i)$ & $(0.6 i, 0.4 i)$ \\
\hline$x_{3}$ & $(-0.7 i,-0.1 i)$ & $(-0.8 i,-0.1 i)$ & $(0.8 i, 0.5 i)$ \\
\hline$x_{4}$ & $(0.6,0.1)$ & $(0.1 i, 0.9 i)$ & $(0.6 i, 0.4 i)$ \\
\hline$x_{5}$ & $(0.2 i, 0.1 i)$ & $(-0.1,-0.6)$ & $(0.3 i, 0.5 i)$ \\
\hline$x_{6}$ & $(0.6 i, 0.1 i)$ & $(0.1 i, 0.9 i)$ & $(0.6 i, 0.4 i)$ \\
\hline
\end{tabular}

TABLE 5: TCIFS $\mathscr{B}(T)$.

\begin{tabular}{cccc}
\hline & $t_{1}$ & $t_{2}$ & $t_{3}$ \\
\hline$x_{1}$ & $(0.1 i, 0.1 i)$ & $(-0.1 i,-0.6 i)$ & $(0.1 i, 0.5 i)$ \\
\hline$x_{2}$ & $(0.4,0.3)$ & $(0.7 i, 0.2 i)$ & $(0.9 i, 0.2 i)$ \\
\hline$x_{3}$ & $(-0.7 i,-0.1 i)$ & $(-0.1 i,-0.3 i)$ & $(0.1 i, 0.1 i)$ \\
\hline$x_{4}$ & $(-0.7 i,-0.1 i)$ & $(-0.1 i,-0.7 i)$ & $(0.8 i, 0.5 i)$ \\
\hline$x_{5}$ & $(0.6,0.1)$ & $(0.1 i, 0.9 i)$ & $(0.6 i, 0.4 i)$ \\
\hline$x_{6}$ & $(0.2 i, 0.1 i)$ & $(-0.1,-0.6)$ & $(0.3 i, 0.5 i)$ \\
\hline
\end{tabular}

TABle 6: Then $k(\mathscr{A}, \mathscr{B})$.

\begin{tabular}{cccc}
\hline & $t_{1}$ & $t_{2}$ & $t_{3}$ \\
\hline$x_{1}$ & $k(\mathscr{A}, \mathscr{B})=.0509$ & $k(\mathscr{A}, \mathscr{B})=.1923$ & $k(\mathscr{A}, \mathscr{B})=.2973$ \\
\hline$x_{2}$ & $k(\mathscr{A}, \mathscr{B})=.3041$ & $k(\mathscr{A}, \mathscr{B})=.6592$ & $k(\mathscr{A}, \mathscr{B})=.6648$ \\
\hline$x_{3}$ & $k(\mathscr{A}, \mathscr{B})=.5000$ & $k(\mathscr{A}, \mathscr{B})=.2544$ & $k(\mathscr{A}, \mathscr{B})=.1334$ \\
\hline$x_{4}$ & $k(\mathscr{A}, \mathscr{B})=.4301$ & $k(\mathscr{A}, \mathscr{B})=.6403$ & $k(\mathscr{A}, \mathscr{B})=.6802$ \\
\hline$x_{5}$ & $k(\mathscr{A}, \mathscr{B})=.1360$ & $k(\mathscr{A}, \mathscr{B})=.5508$ & $k(\mathscr{A}, \mathscr{B})=.4204$ \\
\hline$x_{6}$ & $k(\mathscr{A}, \mathscr{B})=.1360$ & $k(\mathscr{A}, \mathscr{B})=.5581$ & $k(\mathscr{A}, \mathscr{B})=.4204$ \\
\hline
\end{tabular}

Proof. Let $t \in T_{1}$. From Definition 10, $\bar{\mu}_{\mathscr{A}}(x, t)=\mu_{\mathscr{A}}(x, t)$, $\bar{v}_{\mathscr{A}}(x, t)=v_{\mathscr{A}}(x, t), \bar{\mu}_{\mathscr{B}}(x, t)=\mu_{\mathscr{B}}(x, t), \bar{v}_{\mathscr{B}}(x, t)=v_{\mathscr{B}}(x, t)$, and then

(1)

$$
\begin{aligned}
T(\mathscr{A}) & =\sum_{i=1}^{n} \sum_{j=1}^{m}\left(\mu_{\mathscr{A}}^{2}\left(x_{i}, t_{i}\right) e^{i\left(2 \pi-\alpha_{\mathscr{A}}(x)\right)}\right. \\
& \left.+v_{\mathscr{A}}^{2}\left(x_{i}, t_{i}\right) e^{i\left(2 \pi-\beta_{\mathscr{A}}(x)\right)}\right) \\
& =\sum_{i=1}^{n} \sum_{j=1}^{m}\left(v_{\mathscr{A}}^{2}\left(x_{i}, t_{i}\right) e^{i\left(2 \pi-\beta_{\mathscr{A}}(x)\right)}\right. \\
& \left.+\mu_{\mathscr{A}}{ }^{2}\left(x_{i}, t_{i}\right) e^{i\left(2 \pi-\alpha_{\mathscr{A}}(x)\right)}\right)=T(\overline{\mathscr{A}})
\end{aligned}
$$

(2) If $\alpha_{\mathscr{A}}(x, t)=2 \pi$ and $\beta_{\mathscr{A}}(x, t)=2 \pi$

$$
\begin{aligned}
C & (\mathscr{A}, \mathscr{A}) \\
& =\sum_{i=1}^{n} \sum_{j=1}^{m}\left(\mu_{\mathscr{A}}\left(x_{i}, t_{i}\right) e^{i \alpha_{\mathscr{A}}(x, t)} \mu_{\mathscr{A}}\left(x_{i}, t_{i}\right) e^{i \alpha_{\mathscr{A}}(x, t)}\right. \\
& \left.+v_{\mathscr{A}}\left(x_{i}, t_{i}\right) e^{i \beta_{\mathscr{A}}(x, t)} v_{\mathscr{A}}\left(x_{i}, t_{i}\right) e^{i \beta_{\mathscr{A}}(x, t)}\right)
\end{aligned}
$$




$$
\begin{aligned}
& =\sum_{i=1}^{n} \sum_{j=1}^{m}\left(\mu_{\mathscr{A}}{ }^{2}\left(x_{i}, t_{i}\right) e^{2 i \alpha_{\mathscr{A}}(x, t)}\right. \\
& \left.+v_{\mathscr{A}}{ }^{2}\left(x_{i}, t_{i}\right) e^{2 i \beta_{\mathscr{A}}(x, t)}\right) \\
& =\sum_{i=1}^{n} \sum_{j=1}^{m}\left(\mu_{\mathscr{A}}{ }^{2}\left(x_{i}, t_{i}\right) e^{i\left(2 \pi-\alpha_{\mathscr{A}}(x)\right)}\right. \\
& \left.+v_{\mathscr{A}}{ }^{2}\left(x_{i}, t_{i}\right) e^{i\left(2 \pi-\beta_{\mathscr{A}}(x)\right)}\right),=T(\mathscr{A})
\end{aligned}
$$

(3)

$$
\begin{aligned}
C & (\mathscr{A}, \mathscr{B}) \\
& =\sum_{i=1}^{n} \sum_{j=1}^{m}\left(\mu_{\mathscr{A}}\left(x_{i}, t_{i}\right) e^{i \alpha_{\mathscr{A}}(x, t)} \mu_{\mathscr{B}}\left(x_{i}, t_{i}\right) e^{i \alpha_{\mathscr{B}}(x, t)}\right. \\
& \left.+\nu_{\mathscr{A}}\left(x_{i}, t_{i}\right) e^{i \beta_{\mathscr{A}}(x, t)} \nu_{\mathscr{B}}\left(x_{i}, t_{i}\right) e^{i \beta_{\mathscr{B}}(x, t)}\right) \\
& =\sum_{i=1}^{n} \sum_{j=1}^{m}\left(\nu_{\mathscr{B}}\left(x_{i}, t_{i}\right) e^{i \beta_{\mathscr{B}}(x, t)} \nu_{\mathscr{A}}\left(x_{i}, t_{i}\right) e^{i \beta_{\mathscr{A}}(x, t)}\right. \\
& \left.+\mu_{\mathscr{B}}\left(x_{i}, t_{i}\right) e^{i \alpha_{\mathscr{B}}(x, t)} \mu_{\mathscr{A}}\left(x_{i}, t_{i}\right) e^{i \alpha_{\mathscr{A}}(x, t)}\right)=C(\mathscr{B},
\end{aligned}
$$$$
\text { A) }
$$

Theorem 18. Suppose that $\mathscr{A}(T)$ and $\mathscr{B}(T)$ are TCIFSs in the universal $X$ with respect to the time set $T$. Then

(1) if $\alpha_{\mathscr{A}}(x, t)=2 \pi$ and $\beta_{\mathscr{A}}(x, t)=2 \pi$, then $k(\mathscr{A}, \mathscr{B})=1$;

(2) $k(\mathscr{A}, \mathscr{B})=k(\mathscr{B}, \mathscr{A})$;

(3) $0<k(\mathscr{A}, \mathscr{B})<1$.

Proof. (1) Let $\mathscr{A}$ and $\mathscr{B}$ be two TCIFSs defined on the universe of discourse $X=\left\{x_{1}, x_{2}, x_{3}, \ldots, x_{n}\right.$ and the time moments $T=\left\{t_{1}, t_{2}, t_{3}, \ldots, t_{m}\right\}$. The correlation coefficient of $\mathscr{A}$ and $\mathscr{B}$ is given by

$$
k(\mathscr{A}, \mathscr{B})=\frac{C(\mathscr{A}, \mathscr{B})}{\sqrt{T(\mathscr{A}) T(\mathscr{B})}}
$$

If $\mathscr{A}=\mathscr{B}$, then $\sqrt{T(\mathscr{A}) T(\mathscr{B})}=\sqrt{T(\mathscr{A})^{2}}=T(\mathscr{A})$. Then from Proposition 17 (2), one has the following.

If $\alpha_{\mathscr{A}}(x, t)=2 \pi$ and $\beta_{\mathscr{A}}(x, t)=2 \pi$, then $C(\mathscr{A}, \mathscr{A})=$ $T(\mathscr{A})$; then

$$
k(\mathscr{A}, \mathscr{B})=\frac{T(\mathscr{A})}{T(\mathscr{A})}=1
$$

(2) From Proposition $17(3), C(\mathscr{A}, \mathscr{B})=C(\mathscr{B}, \mathscr{A})$. Then

$$
k(\mathscr{A}, \mathscr{B})=\frac{C(\mathscr{B}, \mathscr{A})}{\sqrt{T(\mathscr{B}) T(\mathscr{A})}}=k(\mathscr{B}, \mathscr{A})
$$

(3) We will prove that $k(\mathscr{A}, \mathscr{B})<1$ such that it is evident $0<k(\mathscr{A}, \mathscr{B})$, so suppose that

$$
\begin{aligned}
& \sum_{i=1}^{n} \sum_{j=1}^{m} \mu_{\mathscr{A}}{ }^{2}\left(x_{i}, t_{i}\right) e^{i\left(2 \pi-\beta_{\mathscr{A}}(x)\right)}=\alpha_{1}, \\
& \sum_{i=1}^{n} \sum_{j=1}^{m} \mu_{\mathscr{B}}^{2}\left(x_{i}, t_{i}\right) e^{i\left(2 \pi-\beta_{\mathscr{B}}(x)\right)}=\alpha_{2}, \\
& \sum_{i=1}^{n} \sum_{j=1}^{m} v_{\mathscr{A}}^{2}\left(x_{i}, t_{i}\right) e^{i\left(2 \pi-\beta_{\mathscr{A}}(x)\right)}=\alpha_{3}, \\
& \sum_{i=1}^{n} \sum_{j=1}^{m} v_{\mathscr{B}}^{2}\left(x_{i}, t_{i}\right) e^{i\left(2 \pi-\beta_{\mathscr{B}}(x)\right)}=\alpha_{4} .
\end{aligned}
$$

Then

$$
\begin{aligned}
& T(\mathscr{A}) T(\mathscr{B}) \\
& =\left(\sum _ { i = 1 } ^ { n } \sum _ { j = 1 } ^ { m } \left(\mu_{\mathscr{A}}^{2}\left(x_{i}, t_{i}\right)\right.\right. \\
& \left.\left.\cdot e^{i\left(2 \pi-\beta_{\mathscr{A}}(x)\right)}+v_{\mathscr{A}}^{2}\left(x_{i}, t_{i}\right) e^{i\left(2 \pi-\beta_{\mathscr{A}}(x)\right)}\right)\right) \\
& \times\left(\sum _ { i = 1 } ^ { n } \sum _ { j = 1 } ^ { m } \left(\mu_{\mathscr{B}}^{2}\left(x_{i}, t_{i}\right)\right.\right. \\
& \left.\left.\cdot e^{i\left(2 \pi-\alpha_{\mathscr{B}}(x, t)\right)}+\nu_{\mathscr{B}}^{2}\left(x_{i}, t_{i}\right) e^{i\left(2 \pi-\beta_{\mathscr{B}}(x)\right)}\right),\right) \\
& =\left(\left(\sum_{i=1}^{n} \sum_{j=1}^{m}\left(\mu_{\mathscr{A}}{ }^{2}\left(x_{i}, t_{i}\right) e^{i\left(2 \pi-\beta_{\mathscr{S A}}(x)\right)}\right)\right)+\left(\sum_{i=1}^{n} \sum_{j=1}^{m} v_{\mathscr{A}}{ }^{2}\left(x_{i}, t_{i}\right)\right.\right. \\
& \left.\left.\cdot e^{i\left(2 \pi-\beta_{s f}(x)\right)}\right)\right) \\
& \times\left(\left(\sum_{i=1}^{n} \sum_{j=1}^{m} \mu_{\mathscr{B}}^{2}\left(x_{i}, t_{i}\right)\right.\right. \\
& \left.\left.\cdot e^{i\left(2 \pi-\alpha_{\mathscr{B}}(x, t)\right)}\right)+\left(\sum_{i=1}^{n} \sum_{j=1}^{m} \nu_{\mathscr{B}}^{2}\left(x_{i}, t_{i}\right) e^{i\left(2 \pi-\beta_{\mathscr{B}}(x)\right)}\right)\right)
\end{aligned}
$$

Then

$$
\begin{aligned}
& \sqrt{T(\mathscr{A}) T(\mathscr{B})}=\left[\left(\alpha_{1}+\alpha_{3}\right) \times\left(\alpha_{2}+\alpha_{4}\right)\right]^{1 / 2} \\
& \quad=\left(\alpha_{1}+\alpha_{3}\right)^{1 / 2} \times\left(\alpha_{2}+\alpha_{4}\right)^{1 / 2} \\
& C(\mathscr{A}, \mathscr{B}) \\
& =\left(\left(\sum_{i=1}^{n} \sum_{j=1}^{m}\left(\mu_{\mathscr{A}}\left(x_{i}, t_{i}\right) e^{i \alpha_{\mathscr{S}}(x, t)} \mu_{\mathscr{S}}\left(x_{i}, t_{i}\right) e^{i \alpha_{\mathscr{O}}(x, t)}\right)\right)+\left(\sum_{i=1}^{n} \sum_{j=1}^{m} v_{\mathscr{A}}\left(x_{i}, t_{i}\right)\right.\right. \\
& \left.\left.\cdot e^{i \beta_{\mathscr{A}}(x, t)} v_{\mathscr{B}}\left(x_{i}, t_{i}\right) e^{i \beta_{\mathscr{S}}(x, t)}\right)\right),
\end{aligned}
$$

Then

$$
C(\mathscr{A}, \mathscr{B})=\left(\alpha_{1} \times \alpha_{2}\right)^{1 / 2}+\left(\alpha_{3} \times \alpha_{4}\right)^{1 / 2}
$$

Then 


$$
\begin{aligned}
& k^{2}(\mathscr{A}, \mathscr{B}) \\
& \quad \leq \frac{\alpha_{1} \times \alpha_{2}+2\left(\alpha_{1} \times \alpha_{2} \times \alpha_{3} \times \alpha_{4}\right)^{1 / 2}+\alpha_{3} \times \alpha_{4}}{\left(\alpha_{1}+\alpha_{3}\right) \times\left(\alpha_{2}+\alpha_{4}\right)}
\end{aligned}
$$

But

$$
\begin{aligned}
k^{2}(\mathscr{A}, \mathscr{B})-1 & \leq \frac{\alpha_{1} \times \alpha_{2}+2\left(\alpha_{1} \times \alpha_{2} \times \alpha_{3} \times \alpha_{4}\right)^{1 / 2}+\alpha_{3} \times \alpha_{4}}{\left(\alpha_{1}+\alpha_{3}\right) \times\left(\alpha_{2}+\alpha_{4}\right)}-1 \\
& =\frac{\alpha_{1} \times \alpha_{2}+2\left(\alpha_{1} \times \alpha_{2} \times \alpha_{3} \times \alpha_{4}\right)^{1 / 2}+\alpha_{3} \times \alpha_{4}-\left(\alpha_{1}+\alpha_{3}\right) \times\left(\alpha_{2}+\alpha_{4}\right)}{\left(\alpha_{1}+\alpha_{3}\right) \times\left(\alpha_{2}+\alpha_{4}\right)}=\frac{\left[\left(\alpha_{1} \times \alpha_{4}\right)^{1 / 2}-\left(\alpha_{2} \times \alpha_{3}\right)^{1 / 2}\right]^{2}}{\left(\alpha_{1}+\alpha_{3}\right) \times\left(\alpha_{2}+\alpha_{4}\right)}
\end{aligned}
$$

$$
\leq 0
$$

Hence $k^{2}(\mathscr{A}, \mathscr{B})-1 \leq 0$, and then $k(\mathscr{A}, \mathscr{B}) \leq 1$.

Definition 19. Let $S$ : TICFSs $(X, T) \times \operatorname{TICFSs}(X, T) \longrightarrow$ $[0,1]$ be a function, and let $\mathscr{A}(T), \mathscr{B}(T)$ and $C(T)$ be TCIFSs in the universal $X=\left\{x_{1}, x_{2}, x_{3}, \ldots, x_{n}\right\}$ with respect to the time set $T=\left\{t_{1}, t_{2}, t_{3}, \ldots, t_{m}\right\}$. Then $S(\mathscr{A}, \mathscr{B})$ is said to be the similarity degree between TICFSs $\mathscr{A}$ and $\mathscr{B}$, satisfying the following statements:

(1) $0 \leq S(\mathscr{A}, \mathscr{B}) \leq 1$.

(2) $S(\mathscr{A}, \mathscr{B})=1$ if $\mathscr{A}=\mathscr{B}$.

(3) $S(\mathscr{A}, \mathscr{B})=S(\mathscr{B}, \mathscr{A})$.

(4) If $\mathscr{A} \subseteq \mathscr{B} \subseteq C$. Then $S(\mathscr{A}, C) \leq S(\mathscr{A}, \mathscr{B}), S(\mathscr{A}, C) \leq$ $S(\mathscr{B}, C)$.
Now we can have the following degrees of the similarity between $\mathscr{A}, \mathscr{B}$ and satisfy the conditions from (1) to (4). Let

$$
\begin{aligned}
& S_{\mathscr{A}}(i, j)=\mu_{\mathscr{A}}\left(x_{i}, t_{j}\right) e^{i \alpha_{\mathscr{A}}\left(x_{i}, t_{j}\right)}-v_{\mathscr{A}}\left(x_{i}, t_{i}\right) e^{i \beta_{\mathscr{A}}\left(x_{i}, t_{j}\right)}, \\
& S_{\mathscr{B}}(i, j)=\mu_{\mathscr{B}}\left(x_{i}, t_{j}\right) e^{i \alpha_{\mathscr{B}}\left(x_{i}, t_{j}\right)}-v_{\mathscr{B}}\left(x_{i}, t_{i}\right) e^{i \beta_{\mathscr{B}}\left(x_{i}, t_{j}\right)}, \\
& \psi_{\mathscr{A}}(i, j) \\
& \quad=\frac{\mu_{\mathscr{A}}\left(x_{i}, t_{j}\right) e^{i \alpha_{\mathscr{A}}\left(x_{i}, t_{j}\right)}+1-\mu_{\mathscr{B}}\left(x_{i}, t_{j}\right) e^{i \alpha_{\mathscr{B}}\left(x_{i}, t_{j}\right)}}{2}, \\
& \psi_{\mathscr{B}}(i, j) \\
& \quad=\frac{v_{\mathscr{A}}\left(x_{i}, t_{i}\right) e^{i \beta_{\mathscr{A}}\left(x_{i}, t_{j}\right)}+1-v_{\mathscr{B}}\left(x_{i}, t_{i}\right) e^{i \beta_{\mathscr{B}}\left(x_{i}, t_{j}\right)}}{2}
\end{aligned}
$$

$$
\begin{aligned}
& S_{1}(\mathscr{A}, \mathscr{B})=1-\frac{1}{2 m n} \sum_{i=1}^{n} \sum_{j=1}^{m}\left|S_{\mathscr{A}}(i, j)-S_{\mathscr{B}}(i, j)\right|, \\
& S_{2}(\mathscr{A}, \mathscr{B})=1-\frac{1}{2 m n} \sum_{i=1}^{n} \sum_{j=1}^{m}\left|\mu_{\mathscr{A}}\left(x_{i}, t_{j}\right) e^{i \alpha_{\mathscr{S}}\left(x_{i}, t_{j}\right)}-\mu_{\mathscr{B}}\left(x_{i}, t_{j}\right) e^{i \alpha_{\mathscr{B}}\left(x_{i}, t_{j}\right)}\right|+\left|v_{\mathscr{A}}\left(x_{i}, t_{i}\right) e^{i \beta_{\mathscr{S A}}\left(x_{i}, t_{j}\right)}-v_{\mathscr{B}}\left(x_{i}, t_{i}\right) e^{i \beta_{\mathscr{B}}\left(x_{i}, t_{j}\right)}\right|, \\
& S_{0}(\mathscr{A}, \mathscr{B}) \\
& =1-\frac{1}{\sqrt{2 m n}} \sum_{i=1}^{n} \sum_{j=1}^{m} \sqrt{\left(\mu_{\mathscr{A}}\left(x_{i}, t_{j}\right) e^{i \alpha_{\mathscr{S}}\left(x_{i}, t_{j}\right)}-\mu_{\mathscr{B}}\left(x_{i}, t_{j}\right) e^{i \alpha_{\mathscr{B}}\left(x_{i}, t_{j}\right)}\right)^{2}+\left(v_{\mathscr{A}}\left(x_{i}, t_{i}\right) e^{i \beta_{\mathscr{S}}\left(x_{i}, t_{j}\right)}-v_{\mathscr{B}}\left(x_{i}, t_{i}\right) e^{i \beta_{\mathscr{B}}\left(x_{i}, t_{j}\right)}\right)^{2}} \\
& S_{3}(\mathscr{A}, \mathscr{B})=1-\frac{1}{\sqrt[n]{2 m n}} \sqrt{\sum_{i=1}^{n} \sum_{j=1}^{m}\left|\psi_{\mathscr{A}}(i, j)-\psi_{\mathscr{B}}(i, j)\right|^{y}}, \quad 1 \leq y<+\infty, \\
& S_{4}(\mathscr{A}, \mathscr{B})
\end{aligned}
$$

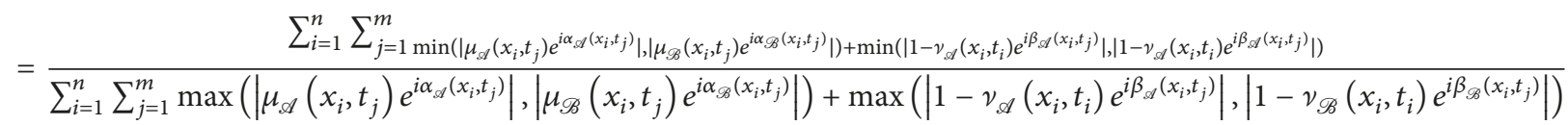

From a comparison between similarity measures $S_{0}(\mathscr{A}, \mathscr{B})$, $S_{1}(\mathscr{A}, \mathscr{B}), S_{2}(\mathscr{A}, \mathscr{B}), S_{3}(\mathscr{A}, \mathscr{B}), S_{4}(\mathscr{A}, \mathscr{B})$, we give the following example.
Example 20. Suppose that $\mathscr{A}(T)$ and $\mathscr{B}(T)$ is TCIFSs defined on $X=\left\{x_{1}, x_{2}, x_{3}, x_{4}, x_{5}, x_{6}\right\}$ with respect to the time set $T=\left\{t_{1}, t_{2}, t_{3}, t_{4}, t_{5}, t_{6}\right\}$. The details of a TCIFS $\mathscr{A}(T)$ 
TABLE 7: TCIFS $\mathscr{A}(T)$.

\begin{tabular}{ccccccc}
\hline & $t_{1}$ & $t_{2}$ & $t_{3}$ & $t_{4}$ & $t_{5}$ & $t_{6}$ \\
\hline$x_{1}$ & $(0.2 i, 0.3 i)$ & $(-0.1 i,-0.3 i)$ & $(0.3 i, 0.5 i)$ & $(0.2 i, 0.1 i)$ & $(0.2 i, 0.1 i)$ & $(0.2 i, 0.1 i)$ \\
\hline$x_{2}$ & $(0.6,0.1)$ & $(0.1 i, 0.9 i)$ & $(0.6 i, 0.4 i)$ & $(0.1 i, 0.1 i)$ & $(-0.1,-0.6)$ & $(0.3,0.5)$ \\
\hline$x_{3}$ & $(-0.7 i,-0.1 i)$ & $(-0.8 i,-0.1 i)$ & $(0.8 i, 0.5 i)$ & $(0.2,0.1)$ & $(0.1 i, 0.9 i)$ & $(0.6 i, 0.4 i)$ \\
\hline$x_{4}$ & $(0.6,0.1)$ & $(0.1 i, 0.9 i)$ & $(0.6 i, 0.4 i)$ & $(-0.7 i,-0.1 i)$ & $(-0.1 i,-0.7 i)$ & $(0.8 i, 0.5 i)$ \\
\hline$x_{5}$ & $(0.2 i, 0.1 i)$ & $(-0.1,-0.6)$ & $(0.3 i, 0.5 i)$ & $(0.2 i, 0.1 i)$ & $(0.2 i, 0.1 i)$ & $(0.2 i, 0.1 i)$ \\
\hline$x_{6}$ & $(0.6 i, 0.1 i)$ & $(0.1 i, 0.9 i)$ & $(0.6 i, 0.4 i)$ & $(0.1 i, 0.2 i)$ & $(-0.1,-0.6)$ & $(0.3,0.5)$ \\
\hline
\end{tabular}

TABLE 8: TCIFS $\mathscr{B}(T)$.

\begin{tabular}{ccccccc}
\hline & $t_{1}$ & $t_{2}$ & $t_{3}$ & $t_{4}$ & $t_{5}$ & $t_{6}$ \\
\hline$x_{1}$ & $(0.1 i, 0.1 i)$ & $(-0.1 i,-0.6 i)$ & $(0.1 i, 0.5 i)$ & $(0.2 i, 0.1 i)$ & $(0.2,0.1)$ & $(0.2 i, 0.1 i)$ \\
\hline$x_{2}$ & $(0.4,0.3)$ & $(0.7 i, 0.2 i)$ & $(0.9 i, 0.2 i)$ & $(0.1,0.1 i)$ & $(0.1 i, 0.6 i)$ & $(0.1,0.2)$ \\
\hline$x_{3}$ & $(-0.7 i,-0.1 i)$ & $(-0.1 i,-0.3 i)$ & $(0.1 i, 0.1 i)$ & $(0.1 i, 0.1)$ & $(0.1 i, 0.8 i)$ & $(0.1 i, 0.4 i)$ \\
\hline$x_{4}$ & $(-0.7 i,-0.1 i)$ & $(-0.1 i,-0.7 i)$ & $(0.8 i, 0.5 i)$ & $(0.6,0.1)$ & $(0.1 i, 0.9 i)$ & $(0.6 i, 0.4 i)$ \\
\hline$x_{5}$ & $(0.6,0.1)$ & $(0.1 i, 0.9 i)$ & $(0.6 i, 0.4 i)$ & $(0.4 i, 0.1 i)$ & $(0.2 i, 0.1 i)$ & $(0.1 i, 0.1 i)$ \\
\hline$x_{6}$ & $(0.2 i, 0.1 i)$ & $(-0.1,-0.6)$ & $(0.3 i, 0.5 i)$ & $(0.3 i, 0.1 i)$ & $(0.2 i, 0.1 i)$ & $(0.2 i, 0.1 i)$ \\
\hline
\end{tabular}

are explained in Table 7 , Table 8 explained TCIFS $\mathscr{B}(T)$, and Table 9 explained a comparison between similarity measures $S_{0}(\mathscr{A}, \mathscr{B}), S_{1}(\mathscr{A}, \mathscr{B}), S_{2}(\mathscr{A}, \mathscr{B}), S_{3}(\mathscr{A}, \mathscr{B}), \quad S_{4}(\mathscr{A}$, $\mathscr{B})$ TCIFS $\mathscr{A}(T)$, and TCIFS $\mathscr{B}(T)$.

\section{Similarity Measures between Other Extensions of Temporal Complex Intuitionistic Fuzzy Set}

The following definition extend the method proposed by Chaira [12] for intuitionistic fuzzy set based on the Sugeno [13] and Omar [10] intuitionistic fuzzy generator.

Definition 21. If $\mu_{\mathscr{A}}(x, t) e^{i \alpha_{\mathscr{A}}(x, t)}$ is the degrees of membership function of the element $x \in X$ at the moment $t \in T$, then nonmembership function $\nu_{\mathscr{A}}(x) e^{i \beta_{\mathscr{A}}(x)}=G\left(\mu_{\mathscr{A}}(x, t) e^{i \alpha_{\mathscr{A}}(x, t)}\right)$, where

$$
G\left(\mu_{\mathscr{A}}(x, t) e^{i \alpha_{\mathscr{A}}(x, t)}\right)=\frac{1-\mu_{\mathscr{A}}(x, t) e^{i \alpha_{\mathscr{A}}(x, t)}}{1+\alpha \mu_{\mathscr{A}}(x, t) e^{i \alpha_{\mathscr{A}}(x, t)}},
$$

$$
\alpha>0
$$

And $G(1)=0, G(0)=1$, and by help of the Sugeno [6] intuitionistic fuzzy generator, TCIFS $\mathscr{A}$ is given by

$$
\begin{aligned}
& \mathscr{A}^{\alpha}(T)=\left\{\left((x, t), \mu_{\mathscr{A}}(x, t)\right.\right. \\
& \left.\left.\cdot e^{i \alpha_{\mathscr{A}}(x, t)}, \frac{1-\mu_{\mathscr{A}}(x, t) e^{i \alpha_{\mathscr{A}}(x, t)}}{1+\alpha \mu_{\mathscr{A}}(x, t) e^{i \alpha_{\mathscr{A}}(x, t)}}\right) \mid(x, t) \in X \times T\right\}
\end{aligned}
$$

The hesitation degree of a TCIFS $\mathscr{A}$ is

$$
\pi_{\mathscr{H}^{\alpha}}(x, t)=1-\mu_{\mathscr{A}}(x, t)-\frac{1-\mu_{\mathscr{A}}(x, t) e^{i \alpha_{\mathscr{A}}(x, t)}}{1+\alpha \mu_{\mathscr{A}}(x, t) e^{i \alpha_{\mathscr{A}}(x, t)}}
$$

Example 22. Suppose that $\mathscr{A}(T)$ is TCIFS defined on $X=$ $\left\{x_{1}, x_{2}, x_{3}\right\}$ with respect to the time set $T=\left\{t_{1}, t_{2}, t_{3}\right\}$. The details of a TCIFS $\mathscr{A}(T)$ are explained in Tables 10, 11, and 12, Table 13 explained TCIFS $\mathscr{A}^{1}$ when $\alpha=1$, and Table 14 explained the hesitation degree of a TCIFS $\mathscr{A}$.

If $\alpha=1$, then one has the following (see Tables 13 and 14).

Definition 23. Suppose that $\mathscr{A}(T)$ and $\mathscr{B}(T)$ is TCIFSs in the universal $X=\left\{x_{1}, x_{2}, x_{3}, \ldots, x_{n}\right\}$ with respect to the time set $T=\left\{t_{1}, t_{2}, t_{3}, \ldots, t_{m}\right\}$. Then a cosine similarity measure between $\mathscr{A}(T)$ and $\mathscr{B}(T)$ is proposed as follows:

$$
\begin{aligned}
C_{T} & \left(\mathscr{A}\left(x_{i}, t_{j}\right), \mathscr{B}\left(x_{i}, t_{j}\right)\right) \\
& =\frac{1}{m n}\left(\sum_{i=1}^{n} \sum_{j=1}^{m} \frac{\mu_{\mathscr{A}}\left(x_{i}, t_{i}\right) e^{i \alpha_{\mathscr{A}}\left(x_{i}, t_{j}\right)} \mu_{\mathscr{B}}\left(x_{i}, t_{i}\right) e^{i \alpha_{\mathscr{B}}\left(x_{i}, t_{j}\right)}+v_{\mathscr{A}}\left(x_{i}, t_{i}\right) e^{i \beta_{\mathscr{A}}\left(x_{i}, t_{j}\right)} v_{\mathscr{B}}\left(x_{i}, t_{i}\right) e^{i \beta_{\mathscr{B}}\left(x_{i}, t_{j}\right)}}{\sqrt{\mu_{\mathscr{A}}^{2}\left(x_{i}, t_{i}\right) e^{i\left(2 \pi-\beta_{\mathscr{A}}(x)\right)}+\nu_{\mathscr{A}}^{2}\left(x_{i}, t_{i}\right) e^{i\left(2 \pi-\beta_{\mathscr{A}}(x)\right)}} \sqrt{\mu_{\mathscr{B}}^{2}\left(x_{i}, t_{i}\right) e^{i\left(2 \pi-\alpha_{\mathscr{B}}(x, t)\right)}+\nu_{\mathscr{B}}^{2}\left(x_{i}, t_{i}\right) e^{i\left(2 \pi-\beta_{\mathscr{B}}(x)\right)}}}\right)
\end{aligned}
$$

where $i=1,2,3, \ldots, n ; j=1,2,3, \ldots, m$ 


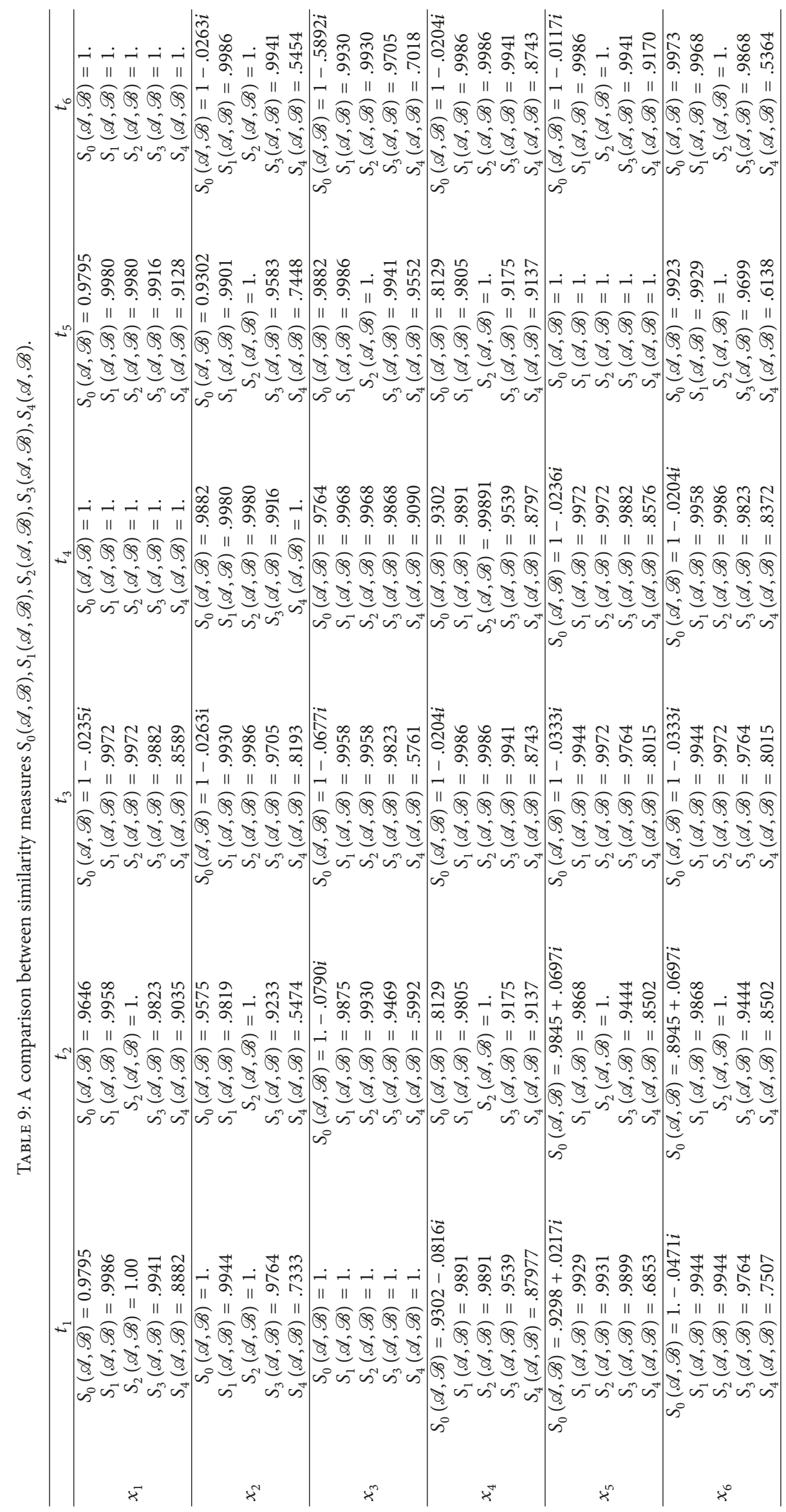


Theorem 24. Suppose that $\mathscr{A}(T)$ and $\mathscr{B}(T)$ are TCIFSs in the universal $X$ with respect to the time set $T$. Then

(1) $C_{T}(\mathscr{A}, \mathscr{B})=1$ if $\mathscr{A}=\mathscr{B}$ and $\alpha_{\mathscr{A}}(x, t)=\beta_{\mathscr{A}}(x, t)=$ $2 \pi$,

(2) $C_{T}(\mathscr{A}, \mathscr{B})=C_{T}(\mathscr{B}, \mathscr{A})$,
(3) $-1 \leq C_{T}(\mathscr{A}, \mathscr{B}) \leq 1$,

(4) if $n=m=1$, then $C_{T}(\mathscr{A}, \mathscr{B})=k(\mathscr{A}, \mathscr{B})$.

Proof. (1) Let $\mathscr{A}$ and $\mathscr{B}$ be two TCIFSs defined on the universe of discourse $X=\left\{x_{1}, x_{2}, x_{3}, \ldots, x_{n}\right\}$ and the time moments $T=\left\{t_{1}, t_{2}, t_{3}, \ldots, t_{m}\right\}$. The cosine similarity measure between $\mathscr{A}(T)$ and $\mathscr{B}(T)$ is given by

$C_{T}(\mathscr{A}, \mathscr{B})$

$$
=\frac{1}{m n}\left(\sum_{i=1}^{n} \sum_{j=1}^{m} \frac{\mu_{\mathscr{A}}\left(x_{i}, t_{i}\right) e^{i \alpha_{\mathscr{A}}(x, t)} \mu_{\mathscr{B}}\left(x_{i}, t_{i}\right) e^{i \alpha_{\mathscr{B}}(x, t)}+v_{\mathscr{A}}\left(x_{i}, t_{i}\right) e^{i \beta_{\mathscr{A}}(x, t)} v_{\mathscr{B}}\left(x_{i}, t_{i}\right) e^{i \beta_{\mathscr{B}}(x, t)}}{\sqrt{\mu_{\mathscr{A}}^{2}\left(x_{i}, t_{i}\right) e^{i\left(2 \pi-\beta_{\mathscr{A}}(x)\right)}+v_{\mathscr{A}}^{2}\left(x_{i}, t_{i}\right) e^{i\left(2 \pi-\beta_{\mathscr{A}}(x)\right)}} \sqrt{\mu_{\mathscr{B}}^{2}\left(x_{i}, t_{i}\right) e^{i\left(2 \pi-\alpha_{\mathscr{B}}(x, t)\right)}+\nu_{\mathscr{B}}^{2}\left(x_{i}, t_{i}\right) e^{i\left(2 \pi-\beta_{\mathscr{B}}(x)\right)}}}\right)
$$

If $\mathscr{A}=\mathscr{B}$ and $\alpha_{\mathscr{A}}(x, t)=\beta_{\mathscr{A}}(x, t)=2 \pi$, then

$C_{T}(\mathscr{A}, \mathscr{B})$

$$
\begin{aligned}
& =\frac{1}{m n}\left(\sum_{i=1}^{n} \sum_{j=1}^{m} \frac{\mu_{\mathscr{A}}{ }^{2}\left(x_{i}, t_{i}\right) e^{i\left(2 \pi-\beta_{\mathscr{A}}(x)\right)}+v_{\mathscr{A}}{ }^{2}\left(x_{i}, t_{i}\right) e^{i\left(2 \pi-\beta_{\mathscr{A}}(x)\right)}}{\mu_{i}{ }^{2} e^{i\left(2 \pi-\beta_{\mathscr{A}}(x)\right)}+v_{\mathscr{A}}{ }^{2}\left(x_{i}, t_{i}\right) e^{i\left(2 \pi-\beta_{\mathscr{A}}(x)\right)}}\right) \\
& =1
\end{aligned}
$$

$$
\begin{aligned}
& C_{T}(\mathscr{A}, \mathscr{B}) \\
& \quad=\frac{1}{m n}\left(\sum_{i=1}^{n} \sum_{j=1}^{m} \frac{\mu_{\mathscr{A}}\left(x_{i}, t_{i}\right) e^{i \alpha_{\mathscr{A}}(x, t)} \mu_{\mathscr{B}}\left(x_{i}, t_{i}\right) e^{i \alpha_{\mathscr{B}}(x, t)}+v_{\mathscr{A}}\left(x_{i}, t_{i}\right) e^{i \beta_{\mathscr{A}}(x, t)} v_{\mathscr{B}}\left(x_{i}, t_{i}\right) e^{i \beta_{\mathscr{B}}(x, t)}}{\left.\sqrt{\mu_{\mathscr{A}}^{2}\left(x_{i}, t_{i}\right) e^{i\left(2 \pi-\beta_{\mathscr{A}}(x)\right)}+v_{\mathscr{A}}^{2}\left(x_{i}, t_{i}\right) e^{i\left(2 \pi-\beta_{\mathscr{A}}(x)\right)}} \sqrt{\mu_{\mathscr{B}}^{2}\left(x_{i}, t_{i}\right) e^{i\left(2 \pi-\alpha_{\mathscr{B}}(x, t)\right)}+v_{\mathscr{B}}^{2}\left(x_{i}, t_{i}\right) e^{i\left(2 \pi-\beta_{\mathscr{B}}(x)\right)}}\right)}\right. \\
& C_{T}(\mathscr{A}, \mathscr{B}) \\
& \quad=\frac{1}{n m}\left(\sum_{j=1}^{m} \sum_{i=1}^{n} \frac{\mu_{\mathscr{B}}\left(x_{i}, t_{i}\right) e^{i \alpha_{\mathscr{B}}(x, t)} \mu_{\mathscr{A}}\left(x_{i}, t_{i}\right) e^{i \alpha_{\mathscr{A}}(x, t)}+v_{\mathscr{B}}\left(x_{i}, t_{i}\right) e^{i \beta_{\mathscr{B}}(x, t)} v_{\mathscr{A}}\left(x_{i}, t_{i}\right) e^{i \beta_{\mathscr{A}}(x, t)}}{\left.\sqrt{\mu_{\mathscr{B}}^{2}\left(x_{i}, t_{i}\right) e^{i\left(2 \pi-\alpha_{\mathscr{B}}(x, t)\right)}+v_{\mathscr{B}}^{2}\left(x_{i}, t_{i}\right) e^{i\left(2 \pi-\beta_{\mathscr{B}}(x)\right)}} \sqrt{\mu_{\mathscr{A}}^{2}\left(x_{i}, t_{i}\right) e^{i\left(2 \pi-\beta_{\mathscr{A}}(x)\right)}+v_{\mathscr{A}}^{2}\left(x_{i}, t_{i}\right) e^{i\left(2 \pi-\beta_{\mathscr{A}}(x)\right)}}\right)}\right) \\
& =C_{T}(\mathscr{B}, \mathscr{A})
\end{aligned}
$$

(3) By the same way in (3) in Theorem 2.1, one has the following.

(4) If $n=m=1$, then

$C_{T}(\mathscr{A}, \mathscr{B})$

$$
=\frac{1}{m n}\left(\sum_{i=1}^{n} \sum_{j=1}^{m} \frac{\mu_{\mathscr{A}}\left(x_{i}, t_{i}\right) e^{i \alpha_{\mathscr{A}}(x, t)} \mu_{\mathscr{B}}\left(x_{i}, t_{i}\right) e^{i \alpha_{\mathscr{A}}(x, t)}+\nu_{\mathscr{A}}\left(x_{i}, t_{i}\right) e^{i \beta_{\mathscr{A}}(x, t)} \nu_{\mathscr{B}}\left(x_{i}, t_{i}\right) e^{i \beta_{\mathscr{A}}(x, t)}}{\sqrt{\mu_{\mathscr{A}}^{2}\left(x_{i}, t_{i}\right) e^{i\left(2 \pi-\beta_{\mathscr{A}}(x)\right)}+\nu_{\mathscr{A}}^{2}\left(x_{i}, t_{i}\right) e^{i\left(2 \pi-\beta_{\mathscr{A}}(x)\right)}} \sqrt{\mu_{\mathscr{B}}^{2}\left(x_{i}, t_{i}\right) e^{i\left(2 \pi-\alpha_{\mathscr{B}}(x, t)\right)}+\nu_{\mathscr{B}}^{2}\left(x_{i}, t_{i}\right) e^{i\left(2 \pi-\beta_{\mathscr{B}}(x)\right)}}}\right)
$$

$C_{T}(\mathscr{A}, \mathscr{B})$

$$
\begin{aligned}
& =\left(\sum_{i=1}^{n} \sum_{j=1}^{m} \frac{\mu_{\mathscr{A}}\left(x_{i}, t_{i}\right) e^{i \alpha_{\mathscr{A}}(x, t)} \mu_{\mathscr{B}}\left(x_{i}, t_{i}\right) e^{i \alpha_{\mathscr{B}}(x, t)}+v_{\mathscr{A}}\left(x_{i}, t_{i}\right) e^{i \beta_{\mathscr{A}}(x, t)} v_{\mathscr{B}}\left(x_{i}, t_{i}\right) e^{i \beta_{\mathscr{B}}(x, t)}}{\sqrt{\mu_{\mathscr{A}}^{2}\left(x_{i}, t_{i}\right) e^{i\left(2 \pi-\beta_{\mathscr{A}}(x)\right)}+\nu_{\mathscr{A}}^{2}\left(x_{i}, t_{i}\right) e^{i\left(2 \pi-\beta_{\mathscr{A}}(x)\right)}} \sqrt{\mu_{\mathscr{B}}^{2}\left(x_{i}, t_{i}\right) e^{i\left(2 \pi-\alpha_{\mathscr{B}}(x, t)\right)}+v_{\mathscr{B}}^{2}\left(x_{i}, t_{i}\right) e^{i\left(2 \pi-\beta_{\mathscr{B}}(x)\right)}}}\right) \\
& =k(\mathscr{A}, \mathscr{B})
\end{aligned}
$$


Definition 25. Suppose that $\mathscr{A}(T)$ and $\mathscr{B}(T)$ is TCIFSs in the universal $X=\left\{x_{1}, x_{2}, x_{3}, \ldots, x_{n}\right\}$ with respect to the time set $T=\left\{t_{1}, t_{2}, t_{3}, \ldots, t_{m}\right\}$. Then, the distance measure of the angle is proposed as follows:

$$
d(\mathscr{A}, \mathscr{B})=\cos ^{-1}\left(C_{T}(\mathscr{A}, \mathscr{B})\right)
$$

Theorem 26. Suppose that $\mathscr{A}(T)$ and $\mathscr{B}(T)$ are TCIFSs in the universal $X$ with respect to the time set $T$. Then

(1) $C_{T}(\mathscr{A}, \mathscr{B})=1$ and $\alpha_{\mathscr{A}}(x, t)=\beta_{\mathscr{A}}(x, t)=2 \pi$; then $d(\mathscr{A}, \mathscr{B})=0$,

(2) $C_{T}(\mathscr{A}, \mathscr{B})=C_{T}(\mathscr{B}, \mathscr{A})$, then $d(\mathscr{A}, \mathscr{B})=d(\mathscr{B}, \mathscr{A})$,

(3) if $-1 \leq C_{T}(\mathscr{A}, \mathscr{B}) \leq 1$, then $d(\mathscr{A}, \mathscr{B}) \geq 0$,

(4) if $\mathscr{A} \subseteq \mathscr{B} \subseteq C$, then $d(\mathscr{A}, C) \leq d(\mathscr{A}, \mathscr{B})+d(\mathscr{B}, C)$.
Proof. (1), (2), and (3) are simple proof.

(4) Let $\mathscr{A}(T), \mathscr{B}(T)$ and $C(T)$ be TCIFSs in the universal $X=\left\{x_{1}, x_{2}, x_{3}, \ldots, x_{n}\right\}$ with respect to the time set $T=$ $\left\{t_{1}, t_{2}, t_{3}, \ldots, t_{m}\right\}$. Then, the distance measure of the angle is proposed as follows:

$$
\begin{aligned}
d_{(i, j)} & \left(\mathscr{A}\left(x_{i}, t_{j}\right), \mathscr{B}\left(x_{i}, t_{j}\right)\right) \\
= & \cos ^{-1}\left(C_{T}\left(\mathscr{A}\left(x_{i}, t_{j}\right), \mathscr{B}\left(x_{i}, t_{j}\right)\right)\right) \\
d_{(i, j)} & \left(\mathscr{A}\left(x_{i}, t_{j}\right), C\left(x_{i}, t_{j}\right)\right) \\
= & \cos ^{-1}\left(C_{T}\left(\mathscr{A}\left(x_{i}, t_{j}\right), C\left(x_{i}, t_{j}\right)\right)\right) \\
d_{(i, j)} & \left(\mathscr{B}\left(x_{i}, t_{j}\right), C\left(x_{i}, t_{j}\right)\right) \\
= & \cos ^{-1}\left(C_{T}\left(\mathscr{B}\left(x_{i}, t_{j}\right), C\left(x_{i}, t_{j}\right)\right)\right)
\end{aligned}
$$

where $i=1,2,3, \ldots, n, j=1,2,3, \ldots, m$, and

$$
\begin{aligned}
& C_{T}\left(\mathscr{A}\left(x_{i}, t_{j}\right), \mathscr{B}\left(x_{i}, t_{j}\right)\right) \\
& =\frac{1}{m n}\left(\sum_{i=1}^{n} \sum_{j=1}^{m} \frac{\mu_{\mathscr{A}}\left(x_{i}, t_{i}\right) e^{i \alpha_{\mathscr{A}}\left(x_{i}, t_{j}\right)} \mu_{\mathscr{B}}\left(x_{i}, t_{i}\right) e^{i \alpha_{\mathscr{B}}\left(x_{i}, t_{j}\right)}+\nu_{\mathscr{A}}\left(x_{i}, t_{i}\right) e^{i \beta_{\mathscr{A}}\left(x_{i}, t_{j}\right)} \nu_{\mathscr{B}}\left(x_{i}, t_{i}\right) e^{i \beta_{\mathscr{B}}\left(x_{i}, t_{j}\right)}}{\sqrt{\mu_{\mathscr{A}}^{2}\left(x_{i}, t_{i}\right) e^{i\left(2 \pi-\beta_{\mathscr{A}}(x)\right)}+\nu_{\mathscr{A}}^{2}\left(x_{i}, t_{i}\right) e^{i\left(2 \pi-\beta_{\mathscr{A}}(x)\right)}} \sqrt{\mu_{\mathscr{B}}^{2}\left(x_{i}, t_{i}\right) e^{i\left(2 \pi-\alpha_{\mathscr{B}}(x, t)\right)}+\nu_{\mathscr{B}}^{2}\left(x_{i}, t_{i}\right) e^{i\left(2 \pi-\beta_{\mathscr{B}}(x)\right)}}}\right) \\
& C_{T}\left(\mathscr{B}\left(x_{i}, t_{j}\right), C\left(x_{i}, t_{j}\right)\right) \\
& =\frac{1}{m n}\left(\sum_{i=1}^{n} \sum_{j=1}^{m} \frac{\mu_{\mathscr{B}}\left(x_{i}, t_{i}\right) e^{i \alpha_{\mathscr{A}}\left(x_{i}, t_{j}\right)} \mu_{C}\left(x_{i}, t_{i}\right) e^{i \alpha_{C}\left(x_{i}, t_{j}\right)}+v_{\mathscr{B}}\left(x_{i}, t_{i}\right) e^{i \beta_{\mathscr{B}}\left(x_{i}, t_{j}\right)} v_{C}\left(x_{i}, t_{i}\right) e^{i \beta_{C}\left(x_{i}, t_{j}\right)}}{\sqrt{\mu_{\mathscr{B}}^{2}\left(x_{i}, t_{i}\right) e^{i\left(2 \pi-\beta_{\mathscr{A}}(x)\right)}+v_{\mathscr{B}}^{2}\left(x_{i}, t_{i}\right) e^{i\left(2 \pi-\beta_{\mathscr{A}}(x)\right)}} \sqrt{\mu_{C}^{2}\left(x_{i}, t_{i}\right) e^{i\left(2 \pi-\alpha_{\mathscr{B}}(x, t)\right)}+v_{C}^{2}\left(x_{i}, t_{i}\right) e^{i\left(2 \pi-\beta_{\mathscr{B}}(x)\right)}}}\right) \\
& C_{T}\left(\mathscr{A}\left(x_{i}, t_{j}\right), C\left(x_{i}, t_{j}\right)\right) \\
& =\frac{1}{m n}\left(\sum_{i=1}^{n} \sum_{j=1}^{m} \frac{\mu_{\mathscr{A}}\left(x_{i}, t_{i}\right) e^{i \alpha_{\mathscr{A}}\left(x_{i}, t_{j}\right)} \mu_{C}\left(x_{i}, t_{i}\right) e^{i \alpha_{C}\left(x_{i}, t_{j}\right)}+v_{\mathscr{A}}\left(x_{i}, t_{i}\right) e^{i \beta_{\mathscr{A}}\left(x_{i}, t_{j}\right)} v_{C}\left(x_{i}, t_{i}\right) e^{i \beta_{C}\left(x_{i}, t_{j}\right)}}{\sqrt{\mu_{\mathscr{A}}^{2}\left(x_{i}, t_{i}\right) e^{i\left(2 \pi-\beta_{\mathscr{A}}(x)\right)}+v_{\mathscr{A}}^{2}\left(x_{i}, t_{i}\right) e^{i\left(2 \pi-\beta_{\mathscr{A}}(x)\right)}} \sqrt{\mu_{C}^{2}\left(x_{i}, t_{i}\right) e^{i\left(2 \pi-\alpha_{C}(x, t)\right)}+v_{C}^{2}\left(x_{i}, t_{i}\right) e^{i\left(2 \pi-\beta_{C}(x)\right)}}}\right)
\end{aligned}
$$

If $\mathscr{A}\left(x_{i}, t_{j}\right) \subseteq \mathscr{B}\left(x_{i}, t_{j}\right) \subseteq C\left(x_{i}, t_{j}\right)$, for each $i=1,2,3, \ldots, n$, $j=1,2,3, \ldots, m$, then

$$
\begin{aligned}
d_{(i, j)} & \left(\mathscr{A}\left(x_{i}, t_{j}\right), \mathscr{B}\left(x_{i}, t_{j}\right)\right) \\
& +d_{(i, j)}\left(\mathscr{B}\left(x_{i}, t_{j}\right), C\left(x_{i}, t_{j}\right)\right) \\
\geq & d_{(i, j)}\left(\mathscr{A}\left(x_{i}, t_{j}\right), C\left(x_{i}, t_{j}\right)\right) .
\end{aligned}
$$

$$
\begin{aligned}
\rho_{T} & \left(\mathscr{A}\left(x_{i}, t_{j}\right), \mathscr{B}\left(x_{i}, t_{j}\right)\right) \\
= & \left(\sum _ { i = 1 } ^ { n } \sum _ { j = 1 } ^ { m } k \left(\mathscr{A}\left(x_{i}, t_{j}\right),\right.\right. \\
& \left.\mathscr{B}\left(x_{i}, t_{j}\right)\right) \\
& \left.\cdot \frac{\mu_{\mathscr{A}}\left(x_{i}, t_{i}\right) e^{i \alpha_{\mathscr{A}}\left(x_{i}, t_{j}\right)} \mu_{\mathscr{B}}\left(x_{i}, t_{i}\right) e^{i \alpha_{\mathscr{B}}\left(x_{i}, t_{j}\right)}+v_{\mathscr{A}}\left(x_{i}, t_{i}\right) e^{i \beta_{\mathscr{A}}\left(x_{i}, t_{j}\right)} \nu_{\mathscr{B}}\left(x_{i}, t_{i}\right) e^{i \beta_{\mathscr{B}}\left(x_{i}, t_{j}\right)}}{\sqrt{\mu_{\mathscr{A}}^{2}\left(x_{i}, t_{i}\right) e^{i\left(2 \pi-\beta_{\mathscr{A}}(x)\right)}+\nu_{\mathscr{A}}^{2}\left(x_{i}, t_{i}\right) e^{i\left(2 \pi-\beta_{\mathscr{A}}(x)\right)}} \sqrt{\mu_{\mathscr{B}}^{2}\left(x_{i}, t_{i}\right) e^{i\left(2 \pi-\alpha_{\mathscr{B}}(x, t)\right)}+v_{\mathscr{B}}^{2}\left(x_{i}, t_{i}\right) e^{i\left(2 \pi-\beta_{\mathscr{B}}(x)\right)}}}\right)
\end{aligned}
$$

Definition 27. Let $\mathscr{A}$ and $\mathscr{B}$ be two TCIFSs defined on the universe of discourse $X=\left\{x_{1}, x_{2}, x_{3}, \ldots, x_{n}\right\}$ and the time moments $T=\left\{t_{1}, t_{2}, t_{3}, \ldots, t_{m}\right\}$. Suppose that $k(\mathscr{A}, \mathscr{B})$ is correlation coefficient of $\mathscr{A}$ and $\mathscr{B}$. Then a weight similarity measure between TCIFSs $\mathscr{A}(T)$ and $\mathscr{B}(T)$ is proposed as follows: 
Table 10: TIFS $\mathscr{A}$.

\begin{tabular}{lccc}
\hline & $t_{1}$ & $t_{2}$ & $t_{3}$ \\
\hline$x_{1}$ & $(0.2,0.1)$ & $(0.1,0.6)$ & $(0.3,0.5)$ \\
\hline$x_{2}$ & $(0.6,0.1)$ & $(0.1,0.9)$ & $(0.6,0.4)$ \\
\hline$x_{3}$ & $(0.7,0.1)$ & $(0.1,0.7)$ & $(0.8,0.5)$ \\
\hline
\end{tabular}

TABLE 11: $\alpha_{\mathscr{A}}(x, t)=\beta_{\mathscr{A}}(x, t)$.

\begin{tabular}{lccc}
\hline & $t_{1}$ & $t_{2}$ & $t_{3}$ \\
\hline$x_{1}$ & $e^{i(\pi / 2)}$ & $e^{i \pi}$ & $e^{i 2 \pi}$ \\
\hline$x_{2}$ & $e^{i 2 \pi}$ & $e^{i(\pi / 2)}$ & $e^{i(\pi / 2)}$ \\
\hline$x_{3}$ & $e^{i(3 \pi / 2)}$ & $e^{i(3 \pi / 2)}$ & $e^{i(\pi / 2)}$ \\
\hline
\end{tabular}

TABLE 12: TCIFS $\mathscr{A}$.

\begin{tabular}{cccc}
\hline & $t_{1}$ & $t_{2}$ & $t_{3}$ \\
\hline$x_{1}$ & $(0.2 i, 0.1 i)$ & $(-0.1,-0.6)$ & $(0.3,0.5)$ \\
\hline$x_{2}$ & $(0.6,0.1)$ & $(0.1 i, 0.9 i)$ & $(0.6 i, 0.4 i)$ \\
\hline$x_{3}$ & $(-0.7 i,-0.1 i)$ & $(-0.1 i,-0.7 i)$ & $(0.8 i, 0.5 i)$ \\
\hline
\end{tabular}

TABLE 13: TCIFS $\mathscr{A}^{1}$.

\begin{tabular}{cccc}
\hline & $t_{1}$ & $t_{2}$ & $t_{3}$ \\
\hline$x_{1}$ & $\left(0.2 i, \frac{1-0.2 i}{1+0.2 i}\right)$ & $(-0.1,1.2)$ & $(0.3,0.53)$ \\
\hline$x_{2}$ & $(0.6,0.25)$ & $\left(0.1 i, \frac{1-0.1 i}{1+0.1 i}\right)$ & $\left(0.6 i, \frac{1-0.6 i}{1+0.6 i}\right)$ \\
\hline$x_{3}$ & $\left(-0.7 i, \frac{1+0.7 i}{1-0.7 i}\right)$ & $\left(-0.1 i, \frac{1+0.1 i}{1-0.1 i}\right.$ & $\left(0.8 i, \frac{1-0.8 i}{1+0.8 i}\right)$ \\
\hline
\end{tabular}

TABLE 14: The hesitation degree of a TCIFS $\mathscr{A}$.

\begin{tabular}{cccc}
\hline & $t_{1}$ & $t_{2}$ & $t_{3}$ \\
\hline$x_{1}$ & 0.133 & -1.2 & 0.16 \\
\hline$x_{2}$ & 0.15 & $1-0.1 i-\frac{1-0.1 i}{1+0.1 i}$ & $1-0.6 i-\frac{1-0.6 i}{1+0.6 i}$ \\
\hline$x_{3}$ & $1-0.7 i-\frac{1+0.7 i}{1-0.7 i}$ & $1-0.1 i-\frac{1-0.1 i}{1+0.1 i}$ & $1-0.8 i-\frac{1-0.8 i}{1+0.8 i}$ \\
\hline
\end{tabular}

And we have the following properties:

(1) $\rho_{T}(\mathscr{A}, \mathscr{B})=1$ then $\mathscr{A}=\mathscr{B}$

(2) $\rho_{T}(\mathscr{A}, \mathscr{B})=\rho_{T}(\mathscr{B}, \mathscr{A})$

(3) $-1 \leq \rho_{T}(\mathscr{A}, \mathscr{B}) \leq 1$

Remark 28. $\rho_{T}\left(\mathscr{A}\left(x_{i}, t_{j}\right), \mathscr{B}\left(x_{i}, t_{j}\right)\right)=C_{T}\left(\mathscr{A}\left(x_{i}, t_{j}\right), \mathscr{B}\left(x_{i}\right.\right.$, $\left.\left.t_{j}\right)\right)$ if

$$
\sum_{i=1}^{n} \sum_{j=1}^{m} k\left(\mathscr{A}\left(x_{i}, t_{j}\right), \mathscr{B}\left(x_{i}, t_{j}\right)\right)=\frac{1}{m n}
$$

From a comparison between similarity measures $C_{T}(\mathscr{A}, \mathscr{B})$, $\rho_{T}(\mathscr{A}, \mathscr{B})$ we give the following example (the same data in Example 16).

Example 29. Suppose that $\mathscr{A}(T)$ and $\mathscr{B}(T)$ is TCIFSs defined on $X=\left\{x_{1}, x_{2}, x_{3}, x_{4}, x_{5}, x_{6}\right\}$ with respect to the time set $T=\left\{t_{1}, t_{2}, t_{3}, t_{4}, t_{5}, t_{6}\right\}$. The details of a TCIFS $\mathscr{A}(T)$ are explained in Table 15, Table 16 explained TCIFS $\mathscr{B}(T)$, and Table 17 explained a comparison between similarity measures between $C_{T}(\mathscr{A}, \mathscr{B}), \rho_{T}(\mathscr{A}, \mathscr{B})$.

4.1. Application in Pattern Recognition and Medical Diagnosis. Let $L=\left\{x_{1}, x_{2}, x_{3}, x_{4}, x_{5}, x_{6}\right\}$ be the set of symptoms of the diseases with respect to the time set $T=\left\{t_{1}, t_{2}, t_{3}, t_{4}, t_{5}, t_{6}\right\}$ and $L_{1}$ be the set of diagnoses. By using the similarity measures $C_{T}\left(L, L_{1}\right)$ we try to discover that the patient may suffer from one from diseases $L$ which have symptoms $x_{1}$ at the time $t_{1}$, and we let $L$ be standard case symptoms of one of diseases (Table 18) and $L_{1}$ be any case (Table 19); Table 20 explained the similarity measures $C_{T}\left(L, L_{1}\right)$ between a standard case $L$ and any case $L_{1}$.

And we define the symptoms of case by Table 19.

Then Table 20 explained the similarity measures $C_{T}\left(L, L_{1}\right)$ between a standard case $L$ and any case $L_{1}$.

When the similarity measures $-1 \leq C_{T}\left(L, L_{1}\right) \leq 1$ are small, then probability that the patient is suffering from the disease $x$ at the time $t$ is big and the conversely is true.

\subsection{Complex Intuitionistic Fuzzy Topology}

Definition 30. An intuitionistic complex fuzzy topology on $X$ is a family $\tau$ of $C I F$-sets in $X$ which satisfies the following properties:

(1) $\overline{1}, \overline{0} \in \tau$,

(2) if $\mathscr{A}, \mathscr{B} \in \tau$, then $\mathscr{A} \cap \mathscr{B} \in \tau$,

(3) if $\mathscr{B}_{i} \in \tau$ for each $i \in \Gamma$, then $\bigcup_{i \in \Gamma} \mathscr{B}_{i} \in \tau$.

Then $(X, \tau)$ is called complex intuitionistic fuzzy topological space. The elements of $\tau$ are called CIFO-sets and the complement of the CIO-sets is called CIFC-sets.

Example 31. Consider $X=\{a, b, c, d\}$. Let $\mathscr{A}, \mathscr{B}$ be a CIOsubset of $X$, as given by

$$
\begin{aligned}
\mathscr{A} & =\left(\frac{0.2 e^{i 1.3 \pi}, 0.4 e^{i 0.5 \pi}}{a}, \frac{1.0 e^{i 1.5 \pi}, 0.0 e^{i 0.5 \pi}}{b},\right. \\
& \left.\frac{0.7 e^{i 0.3 \pi}, 0.2 e^{i 1.5 \pi}}{c}, \frac{0.8 e^{i 1.1 \pi}, 0.1 e^{i 0.7 \pi}}{d}\right), \\
\mathscr{B} & =\left(\frac{0.2 e^{i 0.9 \pi}, 0.3 e^{i 0.4 \pi}}{a}, \frac{0.1 e^{i 0.2 \pi}, 0.3 e^{i 0.9 \pi}}{b},\right. \\
& \left.\frac{0.1 e^{i 0.1 \pi}, 0.2 e^{i 0.5 \pi}}{c}, \frac{0.5 e^{i 0.2 \pi}, 0.1 e^{i 0.5 \pi}}{d}\right) .
\end{aligned}
$$

Then $\tau=\{\overline{0}, \overline{1}, \mathscr{A}, \mathscr{B}, \mathscr{A} \cap \mathscr{B}, \mathscr{A} \cup \mathscr{B}\}$ is an complex intuitionistic fuzzy topology on $X$. 
TABLE 15: TCIFS $\mathscr{A}(T)$.

\begin{tabular}{ccccccc}
\hline & $t_{1}$ & $t_{2}$ & $t_{3}$ & $t_{4}$ & $t_{5}$ & $t_{6}$ \\
\hline$x_{1}$ & $(0.2 i, 0.3 i)$ & $(-0.1 i,-0.3 i)$ & $(0.3 i, 0.5 i)$ & $(0.2 i, 0.1 i)$ & $(0.2 i, 0.1 i)$ & $(0.2 i, 0.1 i)$ \\
\hline$x_{2}$ & $(0.6,0.1)$ & $(0.1 i, 0.9 i)$ & $(0.6 i, 0.4 i)$ & $(0.1 i, 0.1 i)$ & $(-0.1,-0.6)$ & $(0.3,0.5)$ \\
\hline$x_{3}$ & $(-0.7 i,-0.1 i)$ & $(-0.8 i,-0.1 i)$ & $(0.8 i, 0.5 i)$ & $(0.2,0.1)$ & $(0.1 i, 0.9 i)$ & $(0.6 i, 0.4 i)$ \\
\hline$x_{4}$ & $(0.6,0.1)$ & $(0.1 i, 0.9 i)$ & $(0.6 i, 0.4 i)$ & $(-0.7 i,-0.1 i)$ & $(-0.1 i,-0.7 i)$ & $(0.8 i, 0.5 i)$ \\
\hline$x_{5}$ & $(0.2 i, 0.1 i)$ & $(-0.1,-0.6)$ & $(0.3 i, 0.5 i)$ & $(0.2 i, 0.1 i)$ & $(0.2 i, 0.1 i)$ & $(0.2 i, 0.1 i)$ \\
\hline$x_{6}$ & $(0.6 i, 0.1 i)$ & $(0.1 i, 0.9 i)$ & $(0.6 i, 0.4 i)$ & $(0.1 i, 0.2 i)$ & $(-0.1,-0.6)$ & $(0.3,0.5)$ \\
\hline
\end{tabular}

TABLE 16: TCIFS $\mathscr{B}(T)$.

\begin{tabular}{ccccccc}
\hline & $t_{1}$ & $t_{2}$ & $t_{3}$ & $t_{4}$ & $t_{5}$ & $t_{6}$ \\
\hline$x_{1}$ & $(0.1 i, 0.1 i)$ & $(-0.1 i,-0.6 i)$ & $(0.1 i, 0.5 i)$ & $(0.2 i, 0.1 i)$ & $(0.2,0.1)$ & $(0.2 i, 0.1 i)$ \\
\hline$x_{2}$ & $(0.4,0.3)$ & $(0.7 i, 0.2 i)$ & $(0.9 i, 0.2 i)$ & $(0.1,0.1 i)$ & $(0.1 i, 0.6 i)$ & $(0.1,0.2)$ \\
\hline$x_{3}$ & $(-0.7 i,-0.1 i)$ & $(-0.1 i,-0.3 i)$ & $(0.1 i, 0.1 i)$ & $(0.1 i, 0.1)$ & $(0.1 i, 0.8 i)$ & $(0.1 i, 0.4 i)$ \\
\hline$x_{4}$ & $(-0.7 i,-0.1 i)$ & $(-0.1 i,-0.7 i)$ & $(0.8 i, 0.5 i)$ & $(0.6,0.1)$ & $(0.1 i, 0.9 i)$ & $(0.6 i, 0.4 i)$ \\
\hline$x_{5}$ & $(0.6,0.1)$ & $(0.1 i, 0.9 i)$ & $(0.6 i, 0.4 i)$ & $(0.4 i, 0.1 i)$ & $(0.2 i, 0.1 i)$ & $(0.1 i, 0.1 i)$ \\
\hline$x_{6}$ & $(0.2 i, 0.1 i)$ & $(-0.1,-0.6)$ & $(0.3 i, 0.5 i)$ & $(0.3 i, 0.1 i)$ & $(0.2 i, 0.1 i)$ & $(0.2 i, 0.1 i)$ \\
\hline
\end{tabular}

Definition 32. If $(X, \tau)$ is called complex intuitionistic fuzzy topological space, $A \subseteq X$, then the interior of $A$ is defined as the union of all CIFO-subsets of $A$ and it is denoted by $A^{\circ}$. That is, $A^{\circ}$ is the largest CIFO-subset of $A$. The closure of $A$ is defined as the intersection of all CIFC sets containing $A$ and it is denoted by $A^{-}$. That is, $A^{-}$is the smallest CIFC-set containing $A$.

Example 33. Consider $X=\{a, b\}$. Let $\mathscr{A}, \mathscr{B}$ be a $C I F$-subset of $X$, as given by

$$
\begin{aligned}
& \mathscr{A}=\left(\frac{0.2 e^{i 0.3 \pi}, 0.4 e^{i 0.5 \pi}}{a}, \frac{0.2 e^{i 0.1 \pi}, 0.1 e^{i 0.7 \pi}}{b}\right), \\
& \mathscr{B}=\left(\frac{0.2 e^{i 0.9 \pi}, 0.3 e^{i 0.4 \pi}}{a}, \frac{0.5 e^{i 0.2 \pi}, 0.1 e^{i 0.5 \pi}}{b}\right) . \\
& C=\left(\frac{0.1 e^{i 0.7 \pi}, 0.2 e^{i 0.1 \pi}}{a}, \frac{0.4 e^{i 0.1 \pi}, 0.1 e^{i 0.1 \pi}}{b}\right)
\end{aligned}
$$

Then $\tau=\{\overline{0}, \overline{1}, \mathscr{A}, \mathscr{B}\}$ is an intuitionistic complex fuzzy topology on $X, C^{\circ}=\overline{0}=\left\{x,\left(0, e^{i(2 \pi)}\right): x \in X\right\}$ and $C^{-}=\overline{1}=\left\{x,\left(e^{i(2 \pi)}, 0\right): x \in X\right\}$.

Definition 34. An intuitionistic complex fuzzy topological space $(X, \tau)$ is said to be extremely disconnected, if the closure of each CIFO-set is CIFO-set.

Definition 35. Let $(X, \tau)$ be an intuitionistic complex fuzzy topological space. A subset $\mathscr{A}$ of $X$ is said to be CIF semiopen set (by short CIFSO-set) (resp., CIF preopen (by short CIPOset), CIF $\alpha$-open (by short CIF $\alpha O$-set), CIF $\beta$-open (by short CI $\beta O$-set), and CIb-open (by short CIbO-set). If $\mathscr{A} \subseteq$ $\mathscr{A}^{\circ-}$ (resp., $\mathscr{A} \subseteq \mathscr{A}^{-\circ}, \mathscr{A} \subseteq \mathscr{A}^{\circ-\circ}, \mathscr{A} \subseteq \mathscr{A}^{-\circ-}$, and $\subseteq \mathscr{A}^{\circ-} \cup$ $\mathscr{A}^{-\circ}$ ), the family of all CISO-set (resp., CIPO-set, CI $\alpha O$-set,
$C I \beta O$-set, and $C I b O$-set) in $X$ is denoted by $C I S O(X)$ (resp., $C I P O(X), C I \alpha O(X)$, and $C I b O(X))$.

The implications between these concepts in the following diagram and the converse are not true in general,

\section{CIFO}

$\Downarrow$

$\begin{array}{ccc}\text { CISFO } & & \text { CIPFO } \\ \Downarrow & & \Downarrow \\ & & \\ & \text { CIF } \alpha O & \\ \Downarrow & \\ \text { CIbO } & \\ \Downarrow & \\ \text { CIF } \beta-O & \end{array}$

\section{Conclusion}

In this paper we introduced and studied a temporal complex intuitionistic fuzzy sets as generalization of complex Atanassov's intuitionistic fuzzy sets by taking the time in the moving of the point; a correlation between two temporal complex intuitionistic fuzzy sets is discussed. A similarity between temporal complex intuitionistic fuzzy sets is main points in the paper as a generalization of the similarity introduced by Omar [10] and Sugeno [13]. We calculate the results by the program Maple 7. Finally we give an applications to know if the patient is suffering from the diseases or not and introduce the main building in a topology by using the same the set. In future research, similarity measures 


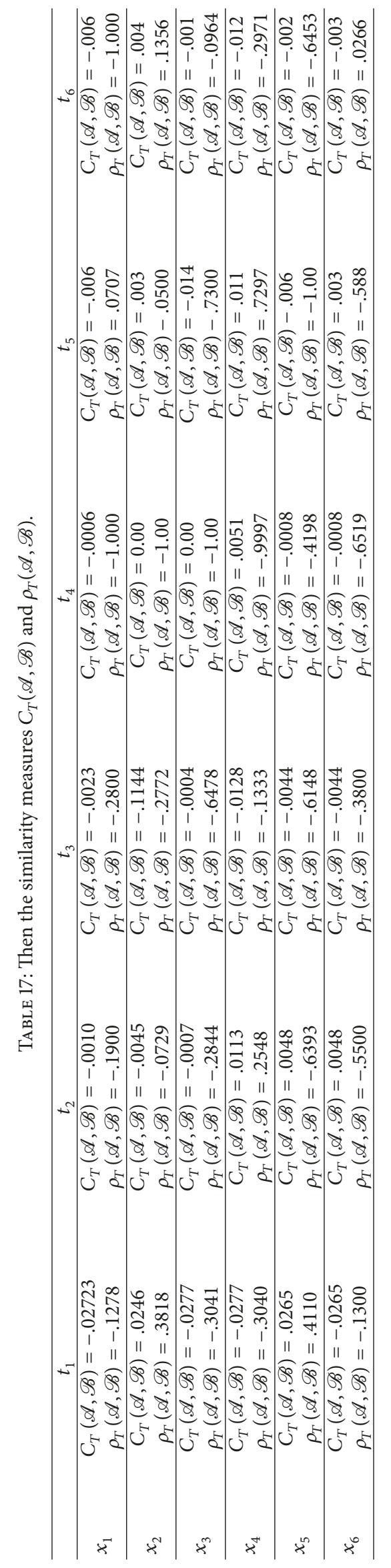


TABLE 18: Standard case symptoms of one of diseases $L$.

\begin{tabular}{cccccrc}
\hline & $t_{1}$ & $t_{2}$ & $t_{3}$ & $t_{4}$ & $t_{5}$ & $t_{6}$ \\
\hline$x_{1}$ & $(0.2 i, 0.3 i)$ & $(-0.1 i,-0.3 i)$ & $(0.3 i, 0.5 i)$ & $(0.2 i, 0.1 i)$ & $(0.2 i, 0.1 i)$ & $(0.2 i, 0.1 i)$ \\
\hline$x_{2}$ & $(0.6,0.1)$ & $(0.1 i, 0.9 i)$ & $(0.6 i, 0.4 i)$ & $(0.1 i, 0.1 i)$ & $(-0.1,-0.6)$ & $(0.3,0.5)$ \\
\hline$x_{3}$ & $(-0.7 i,-0.1 i)$ & $(-0.8 i,-0.1 i)$ & $(0.8 i, 0.5 i)$ & $(0.2,0.1)$ & $(0.1 i, 0.9 i)$ & $(0.6 i, 0.4 i)$ \\
\hline$x_{4}$ & $(0.6,0.1)$ & $(0.1 i, 0.9 i)$ & $(0.6 i, 0.4 i)$ & $(-0.7 i,-0.1 i)$ & $(-0.1 i,-0.7 i)$ & $(0.8 i, 0.5 i)$ \\
\hline$x_{5}$ & $(0.2 i, 0.1 i)$ & $(-0.1,-0.6)$ & $(0.3 i, 0.5 i)$ & $(0.2 i, 0.1 i)$ & $(0.2 i, 0.1 i)$ & $(0.2 i, 0.1 i)$ \\
\hline$x_{6}$ & $(0.6 i, 0.1 i)$ & $(0.1 i, 0.9 i)$ & $(0.6 i, 0.4 i)$ & $(0.1 i, 0.2 i)$ & $(-0.1,-0.6)$ & $(0.3,0.5)$ \\
\hline
\end{tabular}

TABLE 19: The symptoms of case $\mathrm{L}_{1}$.

\begin{tabular}{ccccccc}
\hline & $t_{1}$ & $t_{2}$ & $t_{3}$ & $t_{4}$ & $t_{5}$ & $t_{6}$ \\
\hline$x_{1}$ & $(0.1 i, 0.1 i)$ & $(-0.1 i,-0.6 i)$ & $(0.1 i, 0.5 i)$ & $(0.2 i, 0.1 i)$ & $(0.2,0.1)$ & $(0.2 i, 0.1 i)$ \\
\hline$x_{2}$ & $(0.4,0.3)$ & $(0.7 i, 0.2 i)$ & $(0.9 i, 0.2 i)$ & $(0.1,0.1 i)$ & $(0.1 i, 0.6 i)$ & $(0.1,0.2)$ \\
\hline$x_{3}$ & $(-0.7 i,-0.1 i)$ & $(-0.1 i,-0.3 i)$ & $(0.1 i, 0.1 i)$ & $(0.1 i, 0.1)$ & $(0.1 i, 0.8 i)$ & $(0.1 i, 0.4 i)$ \\
\hline$x_{4}$ & $(-0.7 i,-0.1 i)$ & $(-0.1 i,-0.7 i)$ & $(0.8 i, 0.5 i)$ & $(0.6,0.1)$ & $(0.1 i, 0.9 i)$ & $(0.6 i, 0.4 i)$ \\
\hline$x_{5}$ & $(0.6,0.1)$ & $(0.1 i, 0.9 i)$ & $(0.6 i, 0.4 i)$ & $(0.4 i, 0.1 i)$ & $(0.2 i, 0.1 i)$ & $(0.1 i, 0.1 i)$ \\
\hline$x_{6}$ & $(0.2 i, 0.1 i)$ & $(-0.1,-0.6)$ & $(0.3 i, 0.5 i)$ & $(0.3 i, 0.1 i)$ & $(0.2 i, 0.1 i)$ & $(0.2 i, 0.1 i)$ \\
\hline
\end{tabular}

TABLE 20: Similarity measures $C_{T}\left(L, L_{1}\right)$.

\begin{tabular}{ccccccc}
\hline & $t_{1}$ & $t_{2}$ & $t_{3}$ & $t_{4}$ & $t_{5}$ & $t_{6}$ \\
\hline$x_{1}$ & $C_{T}\left(L, L_{1}\right)=-.02723$ & $C_{T}\left(L, L_{1}\right)=-.0010$ & $C_{T}\left(L, L_{1}\right)=-.0023$ & $C_{T}\left(L, L_{1}\right)=-.0006$ & $C_{T}\left(L, L_{1}\right)=-.006$ & $C_{T}\left(L, L_{1}\right)=-.006$ \\
\hline$x_{2}$ & $C_{T}\left(L, L_{1}\right)=.0246$ & $C_{T}\left(L, L_{1}\right)=-.0045$ & $C_{T}\left(L, L_{1}\right)=-.1144$ & $C_{T}\left(L, L_{1}\right)=0.00$ & $C_{T}\left(L, L_{1}\right)=.003$ & $C_{T}\left(L, L_{1}\right)=.004$ \\
\hline$x_{3}$ & $C_{T}\left(L, L_{1}\right)=-.0277$ & $C_{T}\left(L, L_{1}\right)=-.0007$ & $C_{T}\left(L, L_{1}\right)=-.0004$ & $C_{T}\left(L, L_{1}\right)=0.00$ & $C_{T}\left(L, L_{1}\right)=-.014$ & $C_{T}\left(L, L_{1}\right)=-.001$ \\
\hline$x_{4}$ & $C_{T}\left(L, L_{1}\right)=-.0277$ & $C_{T}\left(L, L_{1}\right)=.0113$ & $C_{T}\left(L, L_{1}\right)=-.0128$ & $C_{T}\left(L, L_{1}\right)=.0051$ & $C_{T}\left(L, L_{1}\right)=.011$ & $C_{T}\left(L, L_{1}\right)=-.012$ \\
\hline$x_{5}$ & $C_{T}\left(L, L_{1}\right)=.0265$ & $C_{T}\left(L, L_{1}\right)=.0048$ & $C_{T}\left(L, L_{1}\right)=-.0044$ & $C_{T}\left(L, L_{1}\right)=-.0008$ & $C_{T}\left(L, L_{1}\right)-.006$ & $C_{T}\left(L, L_{1}\right)=-.002$ \\
\hline$x_{6}$ & $C_{T}\left(L, L_{1}\right)=-.0265$ & $C_{T}\left(L, L_{1}\right)=.0048$ & $C_{T}\left(L, L_{1}\right)=-.0044$ & $C_{T}\left(L, L_{1}\right)=-.0008$ & $C_{T}\left(L, L_{1}\right)=.003$ & $C_{T}\left(L, L_{1}\right)=-.003$ \\
\hline
\end{tabular}

between temporal complex multifuzzy soft and applications in engineering, medical, physics, and automobiles will be studied.

\section{Data Availability}

No data were used to support this study.

\section{Conflicts of Interest}

The author declares no conflicts of interest.

\section{Authors' Contributions}

All authors contributed equally.

\section{Acknowledgments}

Sayer Obaid Alharbi thanks Deanship of Scientific Research (DSR) for providing excellent research facilities. The publication costs of this article were partially covered by the Estonian Academy of Sciences.

\section{References}

[1] L. A. Zadeh, "Fuzzy sets," Information and Computation, vol. 8, pp. 338-353, 1965.

[2] K. T. Atanassov, "Intuitionistic fuzzy sets," Fuzzy Sets and Systems, vol. 20, no. 1, pp. 87-96, 1986.

[3] N. Palaniappan and R. Srinivasan, "Applications of intuitionistic fuzzy sets of root type in image processing," in Proceedings of the NAFIPS 2009 - 2009 Annual Meeting of the North American Fuzzy Information Processing Society, pp. 1-5, Cincinnati, OH, USA, June 2009.

[4] E. Szmidt and J. Kacprzyk, "An application of intuitionistic fuzzy set similarity measures to a multi-criteria decision making problem," in Proceedings of the Artificial Intelligence and Soft Computing-ICAISC, vol. 4029, pp. 314-323, Zakopane, Poland, 2006.

[5] I. K. Vlachos and G. D. Sergiadis, "Intuitionistic fuzzy information-applications to pattern recognition," Pattern Recognition Letters, vol. 28, no. 2, pp. 197-206, 2007.

[6] J. J. Buckley, "Fuzzy complex numbers," Fuzzy Sets and Systems, vol. 33, no. 3, pp. 333-345, 1989.

[7] H. Nguyen, A. Kandel, and V. Kreinovich, "Complex fuzzy sets: towards new foundations," in Proceedings of the Ninth IEEE International Conference on Fuzzy Systems. FUZZ-IEEE 2000. Soft Computing in the Information Age, pp. 1045-1048, San Antonio, TX, USA, 2000. 
[8] D. Ramot, R. Milo, M. Friedman, and A. Kandel, "Complex fuzzy sets," IEEE Transactions on Fuzzy Systems, vol. 10, no. 2, pp. 171-186, 2002.

[9] D. Ramot, M. Friedman, G. Langholz, and A. Kandel, "Complex fuzzy logic," IEEE Transactions on Fuzzy Systems, vol. 11, no. 4, pp. 450-461, 2003.

[10] Omar and G. Maejo, "Similarity measures between temporal intuitionistic fuzzy sets," The International Journal of Science \& Technology, vol. 11, no. 3, pp. 275-284, 2017.

[11] S. Dick, "Toward complex fuzzy logic," IEEE Transactions on Fuzzy Systems, vol. 13, no. 3, pp. 405-414, 2005.

[12] T. Chaira, "Intuitionistic fuzzy segmentation of medical images," IEEE Transactions on Biomedical Engineering, vol. 57, no. 6, pp. 1430-1436, 2010.

[13] M. Sugeno, "Fuzzy measures and fuzzy integrals: a survey," in Fuzzy Automata and Decision Processes, M. M. Gupta, G. N. Saridis, and B. R. Gaines, Eds., pp. 89-102, Noth Holland, New York, NY, USA, 1977.

[14] A. S. Alkouri and A. R. Salleh, "Complex intuitionistic fuzzy sets," in Proceedings of the 2nd International Conference on Fundamental and Applied Sciences 2012, ICFAS 2012, pp. 464470, Malaysia, June 2012.

[15] A. M. Alkouri and A. R. Salleh, "Complex atanassov's intuitionistic fuzzy relation," Abstract and Applied Analysis, vol. 2013, Article ID 287382, 18 pages, 2013.

[16] G. Zhang, T. S. Dillon, K. Y. Cai, J. Ma, and J. Lu, "Operation properties and $\delta$-equalities of complex fuzzy sets," International Journal of Approximate Reasoning, vol. 50, no. 8, pp. 1227-1249, 2009. 


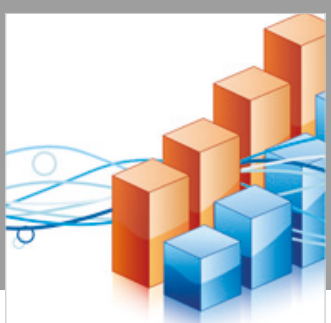

Advances in

Operations Research

\section{-n-m}
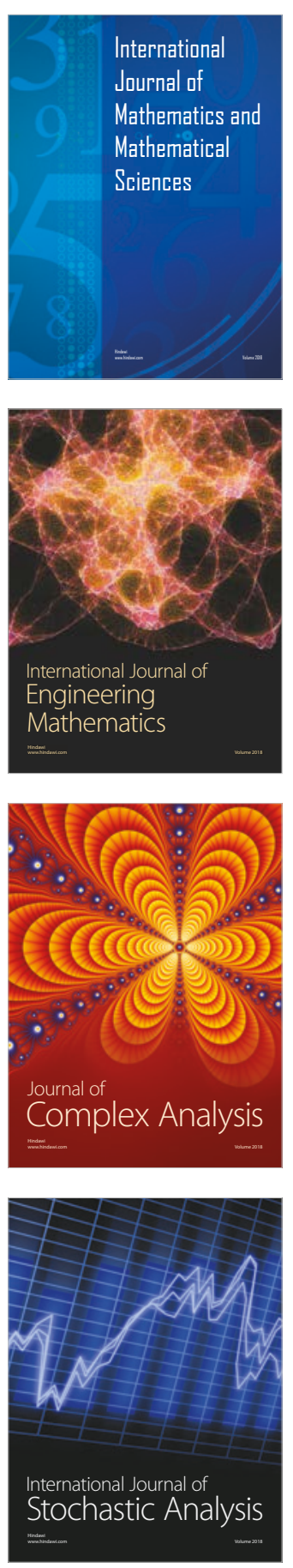
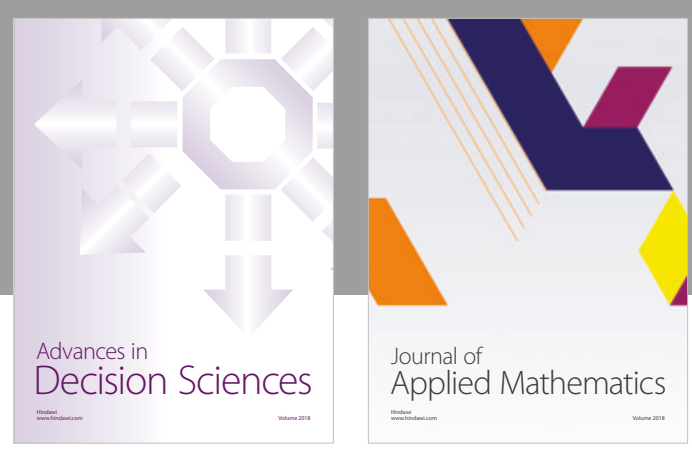

Journal of

Applied Mathematics
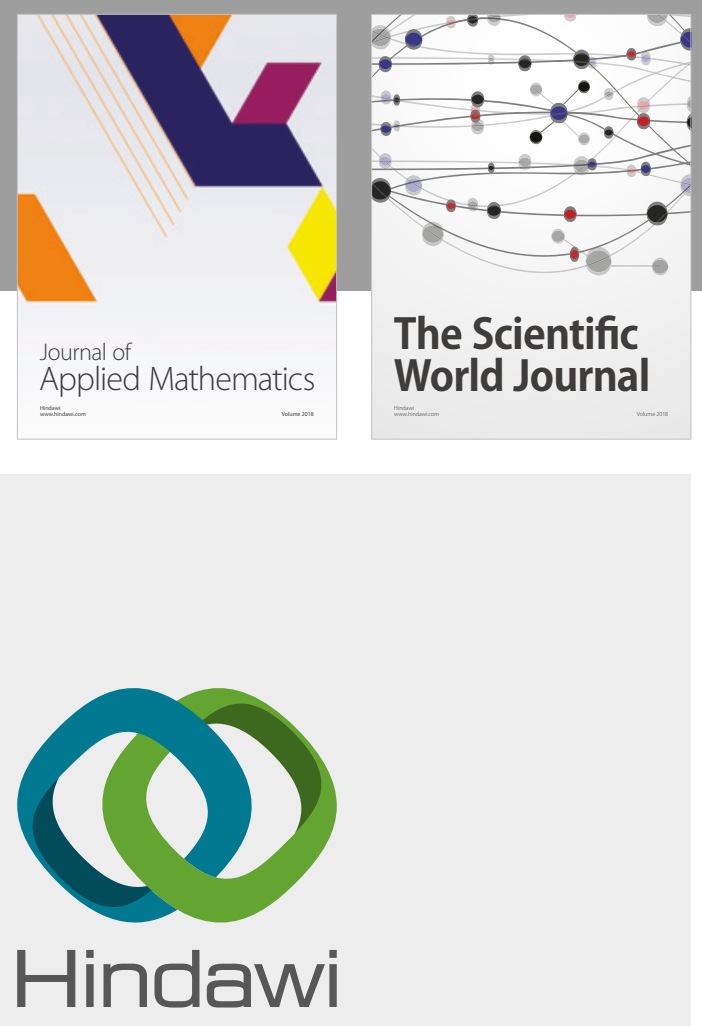

Submit your manuscripts at

www.hindawi.com

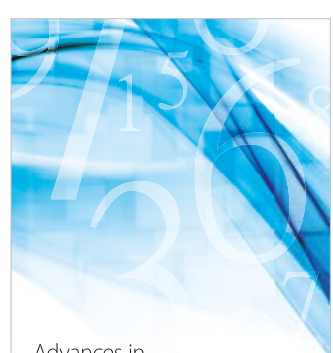

Advances in
Numerical Analysis
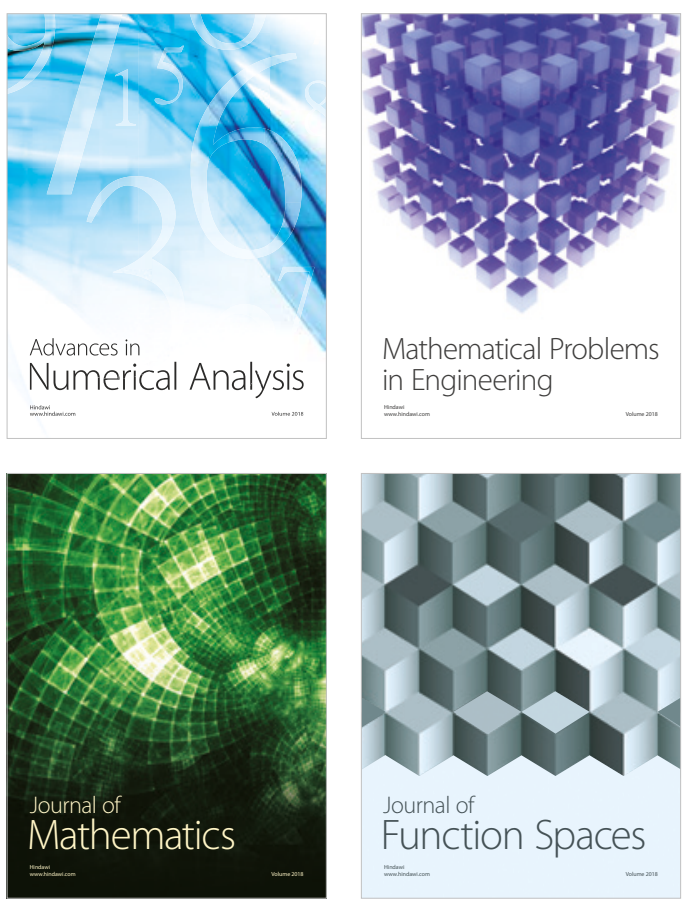

Mathematical Problems in Engineering

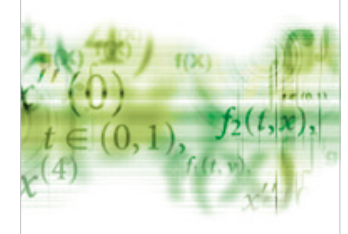

International Journal of

Differential Equations

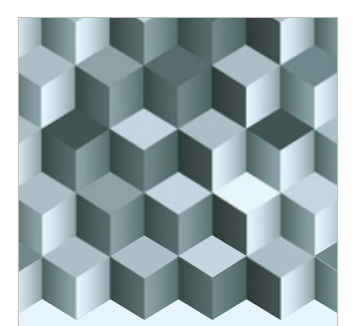

Journal of

Function Spaces

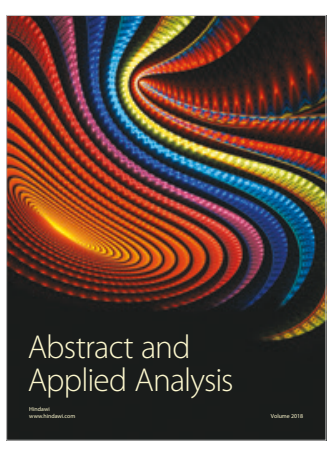

The Scientific

World Journal

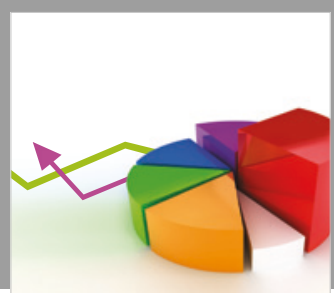

Journal of

Probability and Statistics
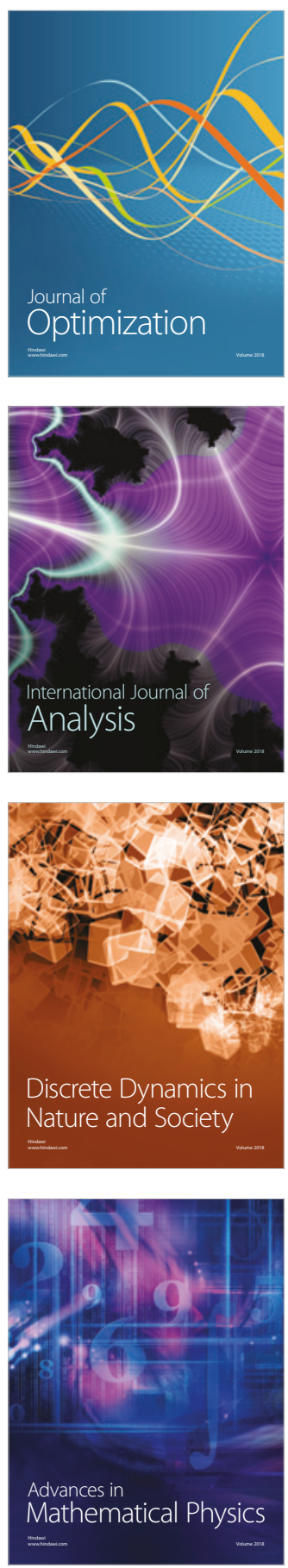